\title{
The Effects of Starburst Activity on Low Surface Brightness Disk Galaxies
}

\author{
Karen O’Neil, G.D. Bothun, \& J. Schombert \\ Dept. of Physics, University of Oregon, Eugene OR, 97403 \\ email:karen@moo.uoregon.edu \\ email:nuts@moo2.uoregon.edu \\ email:js@abyss.uoregon.edu
}

Received _ _ 


\begin{abstract}
Although numerous simulations have been done to understand the effects of intense bursts of star formation on high surface brightness galaxies, few attempts have been made to understand how localized starbursts would affect both the color and surface brightness of low surface brightness (LSB) galaxies. To remedy this, we have run 53 simulations involving bursts of star formation activity on LSB galaxies, varying both the underlying galaxy properties and the parameters describing the starbursts. We discovered that although changing the total color of a galaxy was fairly straightforward, it was virtually impossible to alter a galaxy's central surface brightness and thereby remove it from the LSB galaxy classification without placing a high (and fairly artificial) threshold for the underlying gas density. The primary effect of large amounts of induced star formation was to produce a centralized core (bulge) component which is generally not observed in LSB galaxies. The noisy morphological appearance of LSB galaxies as well as their noisy surface brightness profiles can be reproduced by considering small bursts of star formation that are localized within the disk. The trigger mechanism for such bursts is likely distant/weak tidal encounters. The stability of disk central surface brightness to these periods of star formation argues that the large space density of LSB galaxies at $\mathrm{z}=0$ should hold to substantially higher redshifts.
\end{abstract}

Subject headings: galaxies: evolution; galaxies: formation; galaxies: colors; galaxies: stellar content; galaxies: structure 


\section{Introduction and Background}

Low surface brightness (LSB) galaxies have been systematically under-represented in galaxy surveys due to selection effects whose severity has not been properly appreciated in the past (see reviews by Impey \& Bothun 1997, Bothun, Impey \& McGaugh 1997). Surveys to date (e.g. Bothun et al 1986; Schombert etal 1990; Impey et al 1996; O’Neil et al 1997a) have identified three main classes of LSB galaxies: 1) dwarfs, defined by objects with scale lengths $\leq 1 \mathrm{kpc} ; 2$ ) disk galaxies with scale lengths $1 \leq \alpha \leq 5 \mathrm{kpc}$ and circular velocities in

the range $80-200 \mathrm{~km} / \mathrm{s} ; 3$ ) giant disk galaxies with scale lengths $\geq 5 \mathrm{kpc}$. (As documented by Sprayberry et al (1993), the properties of giant LSB disks are substantially different from those of lower scale length (see also Knezek 1993, Pickering et al 1997).) In this contribution we are only concerned about LSB galaxies that define the second category and which have central surface brightness in the blue fainter than $\mu_{B}(0) \leq 23.0$ mag $\operatorname{arcsec}^{-2}$. For these objects, multicolor photometry, combined with 21-cm observations and H II region spectroscopy are consistent with their having an evolutionary path which branched significantly from that which formed the traditional Hubble sequence (HSB) (i.e. McGaugh \& Bothun 1994; de Blok, Bothun, \& van der Hulst 1995; O’Neil et al 1997b). This suggests a perhaps fundamental difference in star formation history between LSB and HSB disks.

To first order, however, the range of continuum colors between LSB and HSB disks is essentially the same, which broadly means similar stellar populations. The principle differences between the two systems are that 1) LSBs tend to have higher fractional H I contents and 2) at a given circular velocity $\left(V_{c}\right)$ LSBs have a lower stellar abundance (see data in McGaugh 1992; McGaugh 1994). At face value, this suggests that LSBs are less evolved and have had less generations of massive star formation than HSBs. Prima facie evidence for this simple view comes from two principle studies: 1) the measured surface density of H I in LSB disks is 3-6 $M_{\odot} \mathrm{pc}^{-2}$ (Skillman et al 1987; van der Hulst et al 1993; 
de Blok 1997; Pickering et al 1997) - well below the critical density for star formation (e. $g$. Quirk 1972; Kennicutt 1989; Impey \& Bothun 1989); 2) a subsample of objects with velocities $3500-8000 \mathrm{~km} / \mathrm{s}$ drawn from the H II region spectroscopy of McGaugh (1992; 1994) have $\langle\log (\mathrm{O} / \mathrm{H})\rangle=-3.91 \pm 0.30$. This sample consists of 26 individual H II regions in 12 host galaxies and the total observed range is $-4.67<\log (\mathrm{O} / \mathrm{H})<-3.55$. The host galaxies have $V_{c}$ and dynamical masses comparable to $\mathrm{L}_{*}$ HSB disks but an overall metal abundance of $\mathrm{Z}<0.3 Z_{\odot}$.

The surface brightness dependence of the luminosity-metallicity (L-Z) relation for galaxies remains enigmatic. When dwarf galaxies are included (e.g. Skillman 1998), it seems clear that luminosity and not surface brightness is the principle driver. However, Garnett et al (1997) have shown that for disk galaxies, the residuals from the L-Z relation do correlate with local disk surface density. This suggests that while total mass (depth of the potential well) is the principle driver behind metal production, it can be augmented by subsequent density-dependent star formation in the disk. Our contention, based on the data, is that for disk galaxies with scale lengths of $1-5 \mathrm{kpc}$, LSBs have systematically lower abundances than HSBs.

It is possible this low metallicity is a result of a relatively young mean age for these systems as there has been insufficient time to produce many metals. In addition to being consistent with their higher than average fractional gas content, it also helps to explain the very blue colors of some disks. These blue colors are quite difficult to understand in terms of star formation alone, given their low current star formation rates (SFRs) of $\sim 0.1$ $M_{\odot \mathrm{yr}^{-1}}$ (McGaugh 1992; de Blok 1997). Moreover, in $V-I$, LSB disks are often bluer than the most metal-poor globular clusters, showing that the low metallicities alone cannot account for the blue colors (McGaugh \& Bothun 1994). Instead it appears very blue LSB galaxies are among the least evolved objects known. The best example of this is provided 
by UGC 12695, a large, gas-rich LSB which is perhaps the bluest disk in the nearby Universe (O’Neil et al 1998a).

What we wish to explore in this paper is the coupling between LSB and HSB disks. Specifically, the measured SFR fails to produce the observed number of stars in LSB disks (e.g. their total luminosities) by an order of magnitude. This indicates that at times in the past the SFR must have been substantially larger than its current value. Given this, we probe the issue as to whether or not episodic star formation in disk galaxies drives sufficient excursions in surface brightness such that the typical disk galaxy may go through alternating periods of being either LSB or HSB. A priori, we know that this can not be the explanation for the very blue disks. However, a contingent of very red LSB galaxies has been recently discovered (O’Neil, et al 1997a) which can be plausibly identified with faded disks. Between these extremes is a fairly continuous range of LSB galaxy colors, including an important group whose V-I colors indicate an underlying old stellar population but whose $\mathrm{U}-\mathrm{B}$ colors indicate recent star formation in substantial excess of the average past rate (O'Neil, et al 1997a). This mix of galaxy colors makes understanding the effects of starburst on LSB galaxies important to understanding LSB properties and morphology.

If LSB disks have experienced episodic star formation then this begs an obvious question could LSB galaxies undergo significant bursts of star formation and still retain their faint, diffuse appearance? The aim of this paper is to address that question by formulating a $2 \mathrm{D}$ model that explores the effects of increased star formation activity at local places in the LSB disk on its overall color and luminosity profile. In section two we describe our assumptions and underlying methodology. Section three gives our computational results showing how altering both the total mass undergoing starburst and the underlying galaxy properties $\left(\mu(0), \alpha, \mathrm{M}_{\text {gas }} / \mathrm{L}_{\mathrm{T}}\right.$, etc) affect the final galaxy color and surface brightness. Section four discusses potential triggering mechanisms for LSB galaxy starbursts and 
compares the results of our models with the observed LSB galaxy colors.

\section{Components of the Model}

\subsection{Physical Underpinning}

In addition to the differences cited in Section 1 between LSBs and HSBs, de Blok \& McGaugh (1997), based on dynamical data, strongly advocate that LSB disks have fundamentally lower surface mass densities than HSB disks (and LSBs may be more dark matter dominated). This leads to the physically reasonable situation that the density of gas follows the density of stars $\left((\delta \rho / \rho)_{\text {gas }} \propto(\delta \rho / \rho)_{\text {stars }}\right)$. If the production of Giant Molecular Clouds (GMCs) and subsequent massive star formation is density dependent, this apparent physical difference between LSBs and HSBs may directly translate into differences in star formation histories. Certainly those LSB systems measured to date seem to lie below the threshold column density needed for the formation of GMCs.

Studies of LSB disks have found them to be deficient in molecular gas and dust (see Schombert et al 1990; Schombert et al 1992; de Blok \& McGaugh 1997). It should be noted, though, that it is certainly possible that small amounts of $C O$ may have escaped detection due to beam dilution effects. Indeed, its these possible small scale regions of molecular material that may be fueling the 2 - 4 individual H II regions usually observed in LSB disks. The nature of these H II regions shows that stars of at least 50-70 $M_{\odot}$ are present so some massive star formation and metal enrichment is occurring. However, the filling factor of H II regions is very low currently and, given the SFR associated with these

$\mathrm{H}$ II regions, it is not possible that the entire stellar content of LSB disks can be produced in this manner. Hence, if large scale star formation in any galactic disk requires a GMC 
component to the ISM then clearly there must be times when an LSB disk has enough molecular material to generate a burst of star formation. This argues that

$(\delta \rho / \rho)_{\text {gas }} \propto(\delta \rho / \rho)_{\text {stars }}$ is not continuous but must bottom out at some threshold $(\delta \rho / \rho)_{\text {gas }}$ so that there remains an unused reservoir of sufficiently high column density gas that can be converted to molecular material.

Limited observational support for this comes from various 21-cm mapping of LSB systems (e.g. Skillman et al 1987; van der Hulst et al 1993; van Zee et al 1997; Pickering et al 1997) in which central column densities less than $10^{20} \mathrm{~cm}^{-2}$ are not observed. However, we emphasize that a representative sample of LSB disks has not yet been mapped in H I and objects with lower column density may yet appear (or be serendipitously detected in the Parkes Multibeam survey - see Webster et al 1998). Nonetheless, we adopt this gas density threshold for a subset of our models to determine the effects it may have on the starbursts. These particular models, then, are equivalent to allowing any LSB disk in our model to experience elevated levels of star formation if some agent acts to clump the gas regardless of $\mu_{B}(0)$.

\subsection{Our Definition of a Starburst}

The purpose of our model is to explore the effects that brief, intensive, but localized starbursts have on the color and surface brightness profiles of typical LSB galaxies. In particular, we wish to determine if a starburst could, in fact, increase the central surface brightness of an LSB disk and move it out of that domain. At this point, its important to clarify what we mean by "starburst" to avoid confusion later on. Traditionally, starburst galaxies are morphologically distinguished by strong central regions of star formation which typically increase the bolometric luminosity of the host galaxy by a factor of 2-10. Central 
starbursts of this amplitude are usually triggered via galaxy interactions or merging (see Mihos \& Bothun 1998; Smith et al 1996). Heckman et al (1998) have detected a very important attribute of these starburst galaxies, namely, that it is only in very metal poor systems in which most of the flux associated with the starburst escapes the galaxy at UV wavelengths. In metal-normal and metal-rich systems, most of the intrinsic UV flux escapes the galaxy as re-processed Far Infrared radiation. This result strongly increases our expectation that starburst activity in a LSB disk would manifest itself mostly as an increase in the observed UV/blue surface brightness. Since LSB disks are generally observed to inhabit low density environments, the probability of their experiencing a strong tidal encounter during a Hubble time is low (i.e. Bothun et al 1993). Thus we would not expect them to experience strong centrally concentrated star formation and to become true starburst galaxies (see also Salzer 1998). As a result, what we are exploring in this paper are the effects of non-centrally located star formation bursts on the overall properties of an isolated, LSB system. For the moment, we don't care how those bursts might be generated.

\subsection{The Model LSB Galaxy}

We have selected an initial LSB galaxy model based on the mean characteristics of LSB galaxies as a class (O’Neil 1997; McGaugh 1992): 1) no central bulge or bar, 2) low metallicity, 3) a surface density of gas below the canonical threshold for star formation (see Kennicutt 1989). Because the mass density in LSB disks is low (de Blok \& McGaugh 1997), starburst activity can occur in a physical regime where the dynamical time scale is longer than the duration of the starburst. It should be noted that the physics in this situation may be substantially different than when these timescales are reversed in amplitude, such as in the case of ultra-luminous IRAS galaxies (see Downes \& Solomon 1998; Mihos \& Bothun 1998). 
For our models we represent the surface brightness distribution as an exponential, e.g.

$$
\mu(\mathrm{r})=\mu(0)+1.086 \frac{\mathrm{r}}{\alpha}
$$

where $\mu(0)$ is the central surface brightness in mag $\operatorname{arcsec}^{-2}, \alpha$ is the galaxy scale length in $\mathrm{kpc}$, and $\mathrm{r}$ is the radius in kpc. The central surface brightness and scale length were allowed to vary between models, with $\mu_{B}(0)=22.0-24.5 \mathrm{mag} \operatorname{arcsec}^{-2}$ and $\alpha=0.8-$ $18.0 \mathrm{kpc}$, representative of LSB galaxies (i.e. O'Neil, et al 1997b; Pickering, et al 1997; McGaugh \& Bothun 1994). The outer edge of the model galaxy, $r_{T}$, was set at the point where the surface brightness profile drops to $27.0 \mathrm{~B}$ mag $\operatorname{arcsec}^{-2}$.

LSB galaxies typically have a uniform color distribution throughout the disk. This is different than the case for HSB galaxies with similar scale length where significant color gradients are often observed (de Jong 1996). Confusion over the color gradient issue stems from the observations that giant LSB galaxies (those with scale lengths in excess of $5 \mathrm{kpc}$ ) exhibit large color gradients with differences of $0.3-0.5$ mag difference in $\mathrm{B}-\mathrm{R}$ between the inner 2 scale lengths and the outer envelope (Bothun et al 1990; Knezek 1993; de Blok, Bothun, \& van der Hulst 1995; Sprayberry et al 1995). But we are not modeling giant LSB galaxies here. For the LSB disks we therefore assume the underlying colors to be constant between the inner and outer annuli of our defined localized starburst.

The initial mass function (IMF) for each starburst in the model is of the form:

$$
\phi(\mathrm{m})=\mathrm{m}^{-\mathrm{x}} ; \quad \mathrm{x}= \begin{cases}0.25 & m<M_{\odot} \\ 1.35 & M_{\odot}<m<2 M_{\odot} \\ 1.70 & 2 M_{\odot}<m\end{cases}
$$

where the slopes for the IMF were taken from Guiderdoni \& Rocca-Volmerange (1987). 
The IMF is normalized according to $\int_{\mathrm{m}_{1}}^{\mathrm{m}_{\mathrm{u}}} \mathrm{m} \phi(\mathrm{m}) \mathrm{dm}=1$, with $\mathrm{m}_{\text {lower }}=0.55 M_{\odot}$, and $\mathrm{m}_{\text {upper }}$ $=120 M_{\odot}$. To account for the formation of brown dwarfs and planets, $50 \%$ of the star-forming mass is assumed to be converted into non-H-burning objects (Bahcall, Hut, \& Tremaine 1985). If this assumption is incorrect, and more (or less) of the total mass is converted into these objects, then the effects can be compensated for by varying the total amount of the galaxy's mass undergoing starburst.

\subsection{Star Formation in Model Cells}

For the purpose of forming stars, the model galaxy is divided into 30 slices in the radial direction and 30 in the angular direction for a total of 900 cells. The gas available for star formation in a particular cell was determined from the observed range of total gas masses (or fractional gas content) found by de Blok (1997). The underlying gas distribution was assumed to follow the typical HI distribution of LSB galaxies - flat out to $2 \alpha$, and then falling as $1 / r$ (de Blok 1997, Figure 8.2). The radial position of each cell is compared to the HI profile and a gas mass is assigned.

Mihos, de Blok \& McGaugh (1997) have shown that a tidal interaction between a LSB and HSB galaxy of similar mass does not result in a central infall of gas, but instead may cause localized clumping of the gas. To model this type of behavior, we varied where the starburst center lies, letting it range between the true galaxy center and $85 \%$ of the total radius. The strength of the starburst was usually assigned to be $15 \%$ of the galaxy's gas mass. If the total gas mass in the cell containing the initial starburst was greater than, or equal to, the assigned starburst mass, then the starburst was confined to that one cell. Otherwise, the burst radius was allowed to grow to encompass surrounding cells until enough gas mass had been gathered to equal the total burst mass. The starburst in the 
surrounding cells was calculated such that the starburst mass in a neighboring cell, $m(r)$, is given by $m(r)=\frac{c}{r} * m_{\text {initial }}$ where, $m_{\text {initial }}$ is the mass in the initial cell of the starburst, $c$ is a constant which defines the spatial concentration of the burst and $r$ is the distance from the burst center.

This scheme is a variation of that used by Mihos, Richardson, \& Bothun (1991) as, instead of tracking increases in density, we track increases in gas mass in each cell. This is a concession to our ignorance of star formation in a low density environment and whether or not the Schmidt law would be applicable in an ISM in which the molecular gas appears to be either deficient or in very small scale clumps. Phenomenologically then, since we clearly don't understand the physical nature of the ISM in LSBs, it is easier to assess the effects of star formation activity on LSB disk properties by just turning some cell gas mass into stars. This feature of our model, and how the properties of the starburst relate to $c$ and $r$ parameters, is schematically shown in Figures 0 and 1 .

As outlined in Figure 2, the mass used to form stars in a particular cell will depend on how the burst mass is distributed spatially within the galaxy. Since the gas density is low in LSB disks, often the highest stellar masses formed are limited by the low probability of their being drawn from the small, finite gas mass per cell. This is the unique feature of our model. Increases in star formation activity in HSB disks are generally thought to result from the build up of Giant Molecular Clouds (GMCs) and there is sufficient mass to generate a relatively smooth, continuous IMF. However, the low gas densities in LSB disk force us to to consider a situation where the available gas mass is limited and thus the frequency of high mass star formation may be inhibited by small number statistics. Indeed this effect may even be the relevant physics (i.e. Oey \& Clarke 1998; Oey \& Kennicutt 1997). The low gas density requires a significantly larger scale length to reach the gas masses which are usually associated with starburst activity in HSB disks. In some cases, 
this scale length is actually larger than the scale length of the underlying exponential stellar distribution. The physics of star formation in this regime is currently under investigation (O’Neil, Carollo, \& Bothun 1998).

This starved gas mass per cell has the physical effect that our models have difficulty in creating stellar masses $\geq 2.2 M_{\odot}$. Consequently, without a well populated upper main sequence per star formation event, there can be little chemical enrichment and little alteration by the starburst of the pre-existing stellar population's color or surface brightness. This situation is not encountered if we use the threshold approach that fixes a minimum value of $(\delta \rho / \rho)_{\text {gas }}$. In this case, the gas mass per cell which is available will not have as strong of surface brightness dependence and subsequent star formation events will have stronger effects on the overall properties of the LSB disk. We will run both sets of models and discuss which of them are most consistent with the observed properties of LSB disks.

Once the appropriate amount of mass in the cell has been converted into stars using the input efficiency, the total luminosity and color are determined from the IMF. This population is then allowed to evolve using the standard stellar evolution prescriptions. The low metallicity of LSB disks constrains our choice of stellar evolutionary tracks to those with stars having $\mathrm{Z}=0.001$ and $\mathrm{Y}=0.30$ (obtained from Becker 1981; Mengel, et al 1979; Becker \& Iben 1979; Schaller, et al 1992; and Sweigart \& Gross 1978). Once formed in the model, stars were allowed to evolve from the main sequence through their carbon-ignition phase. The original galaxy colors are an input to the model and no color evolution was assumed for this underlying stellar population. Over the duration of the starburst, the evolution of this underlying population is negligible.

As a check that our basic modeling and stellar evolutionary code procedure is valid, we simulated a true starburst by assuming all the gas was in "one" cell and computed, in 
effect, a global change in color and luminosity as a function of percentage of gas mass involved in the burst. These results are shown in Table 1 and are completely consistent with standard starburst models (i.e. Fritze-V. Alvensleben 1998). When applied to the low density ISM of LSB disks, we spread these conditions over many cells. Depending on the choice of model parameters, this produces a range of probabilities of massive star formation. For most choices, this probability is small, unless the gas density threshold approach is used.

\section{Model Results}

\subsection{Overview}

The primary focus of our models is to understand how an occurrence of star formation activity in some local region of a LSB galaxy affects it's overall color and surface brightness profiles. Since LSB galaxies vary greatly in size, luminosity and density, knowing the effects of altering many of the galaxies' other parameters $\left(\mathrm{M} / \mathrm{L}, \mathrm{M}_{\mathrm{gas}} / \mathrm{M}_{\mathrm{T}}\right.$, $\alpha$, etc $)$ is an important step to understanding the starburst process. To this end, a series of models were run altering only one variable at a time. The actual model parameters are given in Table 2, and a summary of the results are given in Table 3. Additionally, to give an idea of the effect the starbursts have on the gas density of the galaxy, a plot showing the before starburst and after gas distributions are shown in Figure 1 for a number of models.

All models were run with $\mathrm{t}_{\text {starburst }}=2$ Myrs and $\Delta \mathrm{t}=1$ Myrs. After 100 Myrs, the stellar population produced by the burst has faded and blended into the underlying stellar population. (See Figure 4 which shows the changes in $U-B$ color with time.) These colors are always expressed in contrast to the original galaxy color, also a variable in Table 2, and 
are plotted as $\Delta(U-B)$ as a function of time. The blue surface brightness profiles and radial color profiles at a timestep of 2 Myrs are shown for every model in Figure $₫$. The colors are plotted relative to the initial galaxy color, $\Delta(U-B)$ as a function of radius. The individual model results plotted in each of these figures are discussed below.

\subsection{Central Surface Brightness}

We begin by considering three LSB galaxies with initial central surface brightness $\mu_{B_{i}}(0)=$ 23.0, 24.0 and $25.0 \mathrm{mag} \operatorname{arcsec}^{-2}$ and we run them through models with and without the HI column threshold. Under the no threshold model, the smallest color change (only -0.04

in $U-B$ ) occurred in the $\mu_{B_{i}}(0)=25.0 \mathrm{mag} \operatorname{arcsec}^{-2}$ model. Recall, that the no threshold model explicitly assumes $(\delta \rho / \rho)_{\text {gas }} \propto(\delta \rho / \rho)_{\text {stars. }}$ In this case, the low assumed gas density plays a very large role. Our procedure places gas mass in each cell, with the amount dependent on the input $\mathrm{M}_{\text {gas }} / \mathrm{M}_{\mathrm{T}}$ and the HI profile. Since $M_{T}$ is smallest in the $\mu_{B_{i}}(0)=$ 25.0 mag $\operatorname{arcsec}^{-2}$ model (because there are less stars and $\alpha$ is constant), there is less gas in each cell for a fixed $\mathrm{M}_{\text {gas }} / \mathrm{M}_{\mathrm{T}}$. This substantially reduces the probability for massive star formation. On the other hand, if we use the threshold model, setting the gas density of each model to the density obtained for a $\mu_{B}(0)=22.0 \mathrm{mag} \operatorname{arcsec}^{-2}$ model, we produce a result that agrees with our intuition. That is, the burst of star formation does produce the most dramatic changes in contrast to a surrounding lower surface brightness stellar population, resulting in the $\mu_{B_{i}}(0)=25.0$ mag $\operatorname{arcsec}^{-2}$ model having the largest color change. These two situations are summarized in Table 1 where the a1-a3 models are for the no threshold case and models 11-14 have the threshold.

To simulate the effects of increasing gas mass, we ran models with and without the gas density threshold but varied the fractional gas content $\mathrm{M}_{\text {gas }} / \mathrm{M}_{\mathrm{T}}$ or the baryonic mass 
fraction $\mathrm{M}_{\mathrm{L}} / \mathrm{M}_{\mathrm{T}}$. Not surprisingly, rising gas masses led to a higher frequency of high mass star formation and bluer overall colors. For instance, for $\mathrm{M}_{\mathrm{T}} / \mathrm{L}_{\mathrm{T}}=10.0 M_{\odot} / L_{\odot}$, a typical value for LSB galaxies (see de Blok \& McGaugh 1997), the change in U-B is -0.20 , and could easily be increased to -0.34 with a small change in $\mathrm{M}_{\text {gas }} / \mathrm{M}_{\mathrm{T}}$ from 0.05 to 0.10 . We also ran an extreme case of the gas density threshold model in which $75 \%$ of the available gas was allowed to undergo a starburst. This model was run with $\mu_{B_{i}}(0)=24.5 \mathrm{mag}$ $\operatorname{arcsec}^{-2}$ and the spatial concentration (c) was set to 50 arcsecond.. The result is the galaxy forms a $\mathrm{r}^{1 / 4}$ (bulge)-type profile. That is, the galaxy behaved the same as a galaxy which collapses or otherwise undergoes a considerable infall of gas to its core.

Establishing the H I column density threshold for models 11-14 carries with it a hidden assumption namely that the galaxy has a significant reservoir of gas, spread throughout its disks, which is available for starburst activity that will elevate the overall surface brightness level. Why is it then that so many of the very bluest LSB galaxies can have $\mu_{B}(0)=24.0-25.0 \mathrm{mag} \operatorname{arcsec}^{-2}$ ? If they have, in fact, been brightened significantly then this implies there is a progenitor population of extremely LSB disks (e.g. $\mu_{B}(0)<25.0$ mag $\operatorname{arcsec}^{-2}$ ) that have large amounts of gas. Its likely that such a population would have been detected in blind 21-cm surveys by now (e.g. Briggs 1990; Spitzak \& Schneider 1998). Furthermore, the red (e.g. $B-V>1.1)$ LSB disks discovered by O'Neil et al (1997a) inhabit the same range of $\mu_{B}(0)$ as the blue LSB disks. Overall, the lack of any $\mu_{B}(0)$ vs. color relation makes any fading scenario unlikely and the gas density threshold scenario problematical. Models without the gas density threshold would tend to preserve this observed non-correlation.

Now we emphasize that, to date, no $\mu_{B_{i}}(0) \leq 23.5$ mag $\operatorname{arcsec}^{-2}$ disk galaxy has yet been mapped in H I so we really can't assess the validity of the gas density threshold model. As a result, the majority of the subsequent models were run with no threshold criteria. If our 
assumption is wrong, and LSB galaxies do indeed have a minimum gas density which is independent of the actual value of $\mu_{B}(0)$, the results can be readily determined by simply adding the appropriate 1 model results to the model in question. We assess the likelihood of the minimum gas density model in Section 4 as the results in Figure 1 clearly indicate that it does lead to substantial changes in $\mu_{B}(0)$.

\subsection{Scale Length}

Starting now with $\mu_{B_{i}}(0)=24.5 \mathrm{mag} \operatorname{arcsec}^{-2}$ and no gas density threshold criteria, models b1 - b4 varied only in the initial value of the scale length, $\alpha$, from $0.8 \mathrm{kpc}$ to 10.0 kpc. The sharpest color changes with time are for the galaxies with the largest scale length, which is well matched by the limited observational data (see de Blok et al 1995) which shows that large scale length LSB disks tend to have relatively strong color gradients. Moreover, the $\alpha=10 \mathrm{kpc}$ model shows a strong blue core. This behavior is highly reminiscent of the class of giant low surface brightness galaxies described in Sprayberry et al (1995), all of which seem to have a pronounced bulge component (like Malin 1 - see Impey \& Bothun 1989) which could be the faded remnant of this blue core.

Changing the scale length is equivalent to altering the total luminosity and mass of the galaxy. However, given our prescription of how the size of the starburst is determined by the need to gather enough mass to reach the variable $\mathrm{m}_{\text {burst }} / \mathrm{m}_{\text {gas }}$ (usually $15 \%$ ), the behavior of the starburst population will vary with the mass within the cells. When the galaxy is large (large $\alpha$ ), then the mass in the cells is high and the starburst is localized. Thus, sufficient numbers of high mass stars are formed per cell, which leads to a bluer integrated color. However, when the galaxy is small, the starburst is spread over a large area and the mass per cell is small and the frequency of forming high mass stars (above 
$\left.2.2 M_{\odot}\right)$ is decreased. The radial color profile also exhibits the effect of a localized versus widespread starburst. The small scale length models have radial color profiles that show the starburst remained near its point of origin (always $2 \alpha$ for these models). The profiles display small blue haloes with red cores. This is reminiscent of the color profiles seen in some nucleated dwarf galaxies in Virgo and Fornax (e.g. Caldwell \& Bothun 1987; Ichikawa et al 1986; Han et al 1998).

\subsection{Starburst Location and Concentration}

In order to understand the effects of the specific location of the starburst on LSB galaxies, we ran a series of simulations, models c1 - c5, varying the radius at which the starburst occurred, $\mathrm{r}_{\text {burst }}$. In model c1, the simulation was run with $15 \%$ of the mass in each cell undergoing starburst, resulting in an even star formation distribution. In models c2 - c5 we let $\mathrm{r}_{\text {burst }} / \mathrm{r}_{\mathrm{T}}$ vary as $0.10,0.30,0.50$, and 0.80 , respectively.

The interpretation of these models is fairly straight-forward, with the starburst activity being very centralized for $\mathrm{r}_{\text {burst }} / \mathrm{r}_{\mathrm{T}} \leq 0.50$. The gas density is high in the core, which produces some high mass star formation at that location. The result is a bluer, higher surface brightness core component to our model LSB disk. The overall change in color $(\Delta(\mathrm{U}-\mathrm{B}))$ was significant, ranging from -0.27 to -0.19 . The change in the surface

brightness profile was also dramatic, with increases of 0.5 to $1.0 \mathrm{~B} \mathrm{mag} \operatorname{arcsec}^{-2}$ in the inner regions. This would have a noticeable impact on the morphological appearance of the galaxy via the rapid development of a bulge or central bar. In general, these central structures are not observed in most LSB disks implying that this mode of star formation does not occur in these systems. As $\mathrm{r}_{\text {burst }} / \mathrm{r}_{\mathrm{T}}$ increased above 0.50 , the starburst activity became non-centralized, producing small bumps in the color/surface brightness profiles 
with amplitudes of -0.08 in $U-B$. The change in their surface brightness profiles was also minimal. Thus, we conclude that the burst location is critical to the evolution of a LSB disk.

The next parameter we varied in our models was the amplitude of the concentration parameter, $c$. Because this parameter is artificial, representing the unknown and highly varying triggering force, we allowed $c$ to range from 1.0" to 100.0". (Recall that c, like the radius, is kept in arcsec for the purpose of modeling. See section 2.2 for more information.) When $c$ was at its lowest value $(c=1.0 "$, model $\mathrm{f} 1)$, the starburst spread across the entire galaxy, and thus inevitably concentrated at the galaxy's core resulting in a sharp change in the galaxy's color profile and a measurable change in the galaxy's inner surface brightness profile (see Figure 4 ). As expected, this scenario comes closest to the global starburst scenario which can indeed transform a LSB disk into a disk of significantly higher surface brightness, and has possible application to the detection of faint blue galaxies at intermediate redshift. We hasten to add, however, that most LSB galaxies at $z=0$ are not in an environment which is conducive to bursts which drive $c=1.0$ (see Bothun et al 1993; Mo, McGaugh, \& Bothun 1994). With $c \geq 5.0 "$, the color profiles show only a localized change at the radius of the initial starburst and the effect on integrated color is substantially reduced (by $0.20-0.30 \mathrm{mag}$ in $U-B$ ). No measurable differences in $\mu_{B}(0)$ could be detected for the $\mathrm{c} \geq 5.0$ " models.

\subsection{Initial Stellar Population}

In an attempt to understand how starbursts would affect galaxies at different stages in their evolution, we altered the colors of the initial stellar population, from very blue $(U-B=-0.20, B-V=0.40, V-I=0.80)$ to the very red $(U-B=0.60$, 
$B-V=1.50, V-I=2.00$ ). The results of this simulation are shown in Figure 1 (models h1-h5). The total change in color between the five models is significant, with the largest change always occurring in the $\mathrm{U}-\mathrm{B}$ color, and the smallest in $\mathrm{V}-\mathrm{I}$. Because the effects of starburst are less significant on galaxies which are already fairly blue, we chose colors slightly redder than the average LSB galaxy color for most of our simulations (i.e. $\mathrm{U}-\mathrm{B}=$ $0.2, \mathrm{~B}-\mathrm{V}=0.8, \mathrm{~V}-\mathrm{I}=1.2)$ to match the colors of new LSB galaxies currently being discovered in CCD surveys (O’Neil et al 1997a).

The starbursts are more easily detected in the radial color profiles for reddest underlying stellar populations. This is simply due to the enhanced contrast bright, blue stars have against an older mix of stars. Additionally, it should be noted that the color change in the red models is far larger in $\mathrm{U}-\mathrm{B}$ than in $\mathrm{V}-\mathrm{I}$, consistent with notion the $V-I$ is a reasonable metallicity indicator due to its lower sensitivity to newly formed stars. Colors that are consistent with the model $U-B$ and $V-I$ values have been detected by O'Neil et al (1997a; i.e. $\mathrm{C} 1-1$ with $\mathrm{U}-\mathrm{B}=0.13$, and $\mathrm{V}-\mathrm{I}=1.20)$.

\subsection{A Flat IMF}

In the no gas density threshold models, the gas density of the system is too low for the formation of many massive stars. We can force this situation to change by considering a flat IMF. This was done using a series of models which put $\mathrm{m}_{\text {lower }}=1.1 M_{\odot}$ in the IMF. These five models were run to mimic the possibilities (and extreme cases) of all the above models. Models j1, j2, and j3 have localized starbursts occurring at $\mathrm{r}=0.60 \mathrm{r}_{T}(\mathrm{j} 1 \& \mathrm{j} 2)$ and $\mathrm{r}=$ $0.10 \mathrm{r}_{T}(\mathrm{j} 3)$, while models $\mathrm{j} 4$ and $\mathrm{j} 5$ have starbursts spread evenly throughout the galaxy.

Models j1 \& j4 used the average values for all of the models, while models j2, j3, \& j5 used an exceptionally high $\mathrm{M}_{\text {gas }} / \mathrm{M}_{T}\left(\mathrm{M}_{\text {gas }} / \mathrm{M}_{T}=0.15\right)$. Figure 4 a shows the surface brightness 
profiles of these models after 2 time steps. What can clearly be seen is that forcing the galaxies to produce high mass stars raises the surface brightness of the burst regions. In all cases, the central surface brightness of these galaxies is raised by at least 0.1 mag $\operatorname{arcsec}^{-2}$, and is raised by $0.4 \mathrm{mag} \operatorname{arcsec}^{-2}$ for model j5. Given the observations that most LSB disks (e.g. those with central surface brightnesses fainter than $23.0 \mathrm{mag} \operatorname{arcsec}^{-2}$ ) are already quite blue, this scenario can't really happen because it would represent the case of an LSB transitioning to a higher surface brightness disk which would have predicted colors much bluer than are generally observed (e.g. galaxies like M101 don't have $B-V \leq 0.3$ ). This, coupled with the observed low metallicity and dust content of these systems, strongly suggests that flat IMF star formation in a metal-poor environment is unlikely to be occurring.

Figure 6 shows the color changes of these galaxies with time. As expected, forcing the galaxies to produce high mass stars also causes significant fluctuations in the galaxy colors with time. Again, these changes would be readily detectable, but considering the large spread in colors (up to -1.33 in $V-I$ ) it may be difficult to determine the true state of a galaxy in this stage. As a last, extreme test of the effects of starbursts on LSB galaxies, we returned $\mathrm{m}_{\text {lower }}$ to $0.55 \mathrm{M}_{\odot}$ and let the c parameter go to an extremely high value (c=50.0") which, in effect, allows as much mass in each cell as was available to undergo starburst. At the same time, $\mathrm{M}_{\text {burst }} / \mathrm{M}_{\text {gas }}$ was allowed to vary from 0.25 (model i1) to 0.75 (model i3), and the total $\mathrm{M}_{\text {gas }} / \mathrm{M}_{\mathrm{T}}$ was varied from $5 \%$ (models i1-i3) through $50 \%$ (model i5). The change in the surface brightness profiles for these models was significant, as was the total change in color $(\Delta(\mathrm{U}-\mathrm{B})=-0.28--0.86 ; \Delta(\mathrm{B}-\mathrm{V})=-0.18--0.88 ; \Delta(\mathrm{V}-\mathrm{I})=-0.10-$ -0.94 ; for models i1 - i5, respectively). It should be noted that these models are unrealistic cases but do serve as valid boundary condition checks of our model procedure. That is, if we tell the model to simulate a global starburst, then it does. 


\section{Star Formation in LSB Galaxies}

\subsection{Can $\mu_{B}(0)$ Significantly Change?}

The motivation for our models is to assess the degree to which disk surface brightness is stable to star formation activity. This issue is important in understanding the space density of galaxies as a function of surface brightness and what the overall evolution of this bivariate luminosity function with redshift might be. We have run two general sets of models, one in which the surface density of gas steadily decreases with decreasing $\mu_{B_{i}}(0)$ and one in which a gas density threshold surface density is established which remains constant as $\mu_{B_{i}}(0)$ continues to decrease. The no gas density threshold models generally have sufficiently low surface gas densities that the formation of stars with mass $\geq 2.2 M_{\odot}$ is inhibited in most of the cells where we have introduced a perturbation (see Figure 3). In this case, although the structural nature of the changed due to the starburst activity (for example, gaining a centralized bulge or core), the measured central disk surface brightness for these galaxies never changed significantly. To verify this result, we re-ran all of the models with $\mathrm{M}_{\text {gas }} / \mathrm{M}_{\mathrm{T}}=10 \%$ and $\mathrm{M}_{\text {burst }} / \mathrm{M}_{\text {gas }}=20 \%$. The results were the same although allowing larger percentages of mass undergo starburst increased the total change in color in each model, there was still no change in the central surface brightness of the galaxies. In fact, once $\mu_{B}(0)$ has been specified for a galaxy, altering that value in any significant way was difficult if not impossible to obtain.

The models with no gas threshold show the robustness of $\mu_{B}(0)$ when star formation is occurring in our model low density environment. To put it another way, whenever we force an intense starburst either by a) raising the gas mass to high levels, b) forcing the burst to be centralized either by varying $\mathrm{r}_{\text {burst }} / \mathrm{r}_{\mathrm{T}}$ or $c$ or $\mathrm{c}$ ) forcing a flat IMF to overcome the cell tendency of not producing high mass stars, we generally produce another structural 
component to the galaxy. The observation that LSB disks, in general, have no bulges, bars or nuclear activity would strongly argue that such a centralized starburst never occurred in these systems. The robustness of $\mu_{B}(0)$ to localized star formation events and/or smaller global events is consistent with the high space density of LSB disks now observed (e.g. McGaugh et al 1995; Sprayberry et al 1997a, 1997b; Dalcanton et al 1997).

As surface brightness is determined by the convolution of the mean luminosity of the stellar population and the average separation between stars, our model results would seem to indicate that changes in mean luminosity are more than compensated for by whatever structural conditions existed during the formation epoch of these disks that allowed for relatively low surface number density of stars (see O'Neil 1998). That is, once the basic structure has been laid down, it would seem that increasing $\mu_{B}(0)$ through modest star formation events is extremely unlikely. Thus, there should be little evolution in the distribution of $\mu_{B}(0)$ as a function of redshift out to modest redshifts $(\mathrm{z} \sim 1)$. This implies that the large space density of LSB disks at $\mathrm{z}=0$ should be preserved out to $\mathrm{z} \sim 1$. The Sloan Digital Sky Survey should ultimately produce a data set that either confirms this expectation or demonstrates that our assumptions about the nature of starburst in LSB disks are wrong.

One of those incorrect assumptions may be the gas density in the no threshold models is simply too low to produce much luminosity per star formation event due to a dearth of massive star formation. Thus the only means of obtaining an increase in the galaxy's disk central surface brightness is to allow a significant increase in gas density within the underlying galaxy. Within our models this can be achieved through decreasing the number of cells within the galaxy and thereby increasing the total mass within each cell. This is equivalent to establishing a threshold column density within 2-3 scale lengths independent of $\mu_{B}(0)$. 
In models $\mathrm{k} 1$ - k4 the galaxy was broken up into only 25 cells, thereby allowing for considerably higher concentrations of mass than was available in all the previous models. The subsequent starburst had bursts strength akin to that in the i models, with $\mathrm{M}_{\text {gas }} / \mathrm{M}_{\mathrm{T}}$ $=5 \%-50 \%, \mathrm{c}=50.0 "$, and $\mathrm{M}_{\text {burst }} / \mathrm{M}_{\text {gas }}=25 \%-50 \%$. Additionally, the starburst was spread evenly throughout the galaxy, in the same manner as model c1. The extreme burst strength was necessary to successfully increase $\mu_{B}(0)$ by a measurable amount, while the even distribution of the starburst was done because of the low resolution of the model since the galaxy was broken into only 5 radial pieces the distinction between having the starburst occur at $0.60 \mathrm{R}_{T}$ and $0.30 \mathrm{R}_{T}$ is minimal.

The resultant surface brightness profiles of models k1 - k4 are given in Figure 4 . What should be immediately noted is that even in the case of model k1, where $25 \%$ of the gas of these artificially condensed galaxies was allowed to undergo starburst, the central surface brightness increased by only $0.5 \mathrm{mag} \operatorname{arcsec}^{-2}$. In order to obtain an increase of $1 \mathrm{mag}$

$\operatorname{arcsec}^{-2}$ in the central disk surface brightness, $50 \%$ of the galaxy's total gas content had to undergo a starburst. Thus, in order to achieve a measurable increase in the central surface brightness, either inordinate amounts of gas must be added to the galaxy's core or a substantial pre-existing reservoir of column density $\sim 10^{20}$ must exist independent of $\mu_{B}(0)$

\subsection{Triggers for Gas Flows}

Is it reasonable to expect a delivery mechanism that could introduce large amounts of gas to the core regions? Internal mechanisms such as bar driven flows seem quite unlikely as LSB disks generally don't have any bars. Stochastic processes, such as those operating in irregular galaxies, probably also do not work since LSB disks are strongly rotationally 
dominated. Thus the only real possibility is tidal interactions. The connection between interacting galaxies and SFRs has been well documented both observationally and through computer simulations. Larson \& Tinsley (1978) showed that the very blue colors of some galaxies could be explained if those galaxies were undergoing tidally triggered bursts of star formation. This mechanism was shown to effectively work in spiral-rich clusters of galaxies (e.g. Bothun \& Schommer 1982) where many HSB spirals are located. More recent studies have shown that gravatationally interacting galaxies have a higher (average) SFR than their more isolated counterparts (i.e. Kennicutt, et al 1987; Bushouse 1986). Additionally, Mihos, et al (1991) used simulations to show that, with high surface brightness (HSB) galaxies, tidal interactions between nearby (distance $=12-36 \mathrm{kpc}$ ) non-merging galaxies can result in starbursts involving $12 \%$ - $24 \%$ of the galaxies' mass (see also Mihos et al 1993; Mihos \& Hernquist 1994, 1996).

External triggers for star formation in LSB galaxies, though, are most likely provided by relatively distant tidal disturbances. HSB disks are generally located in an environment which is favorable for a few strong tidal encounters over a Hubble time. These encounters typically cause a central inflow of gas and often the formation of an inner bar. Increase in star formation in HSB galaxies from tidal interactions is therefore centrally concentrated and an appreciable change occurs at the core of the interacting HSB galaxies. LSB galaxies, though, have been shown to be stable against the growth of bar formation and large scale central inflow of gas during tidal encounters but not necessarily stable against local instabilities. Mihos, de Blok, \& McGaugh (1997) simulated a collision between a HSB and LSB galaxy with similar properties. Soon after the closest approach, the HSB galaxy developed a strong bar which persisted through the end of the simulation and which presumably triggered a central inflow of gas and a strong nuclear starburst. Although being strongly perturbed, the LSB galaxy did not form a bar and therefore would not have undergone a similar central starburst. Instead the encounter excited star formation 
throughout the disk or in locally concentrated regions away from the galaxy's center. It is probable, then, that the more distant tidal encounters LSB galaxies experience result in local, non-centralized instabilities in LSB galaxies, and therefore in the triggering of non-centralized starbursts which we modeled in the previous section.

An important note for this discussion is Vázquez and Scalos's (1989) finding that starbursts do not typically occur during the gas compression stage but in fact occur well after the gas has re-established. In other words, the Vázquez and Scalo model suggests that some disks can have tidally induced star formation well after perihelion. Thus although LSB galaxies are often more isolated than their HSB counterparts (Bothun, et al 1993), this does not preclude the galaxy from being in the midst of tidally induced starbursts. Vázquez and Scalo show starbursts occur $20 \tau_{C}-80 \tau_{C}$ after the initial gas density increase. Here $\tau_{C}$ is the typical collision timescale which is likely quite long $\left(>10^{8} \mathrm{yr}\right)$ for the diffuse LSB galaxies being studied. As many of our detected LSB galaxies are located in the outskirts of spiral rich clusters, the Vázquez and Scalo scenario may well apply.

Identifying galaxies which are currently undergoing or have recently undergone star formation is difficult. As is readily seen in Figure 4 , stochastic star formation could easily produce structural noise and hence one possible manifestation of this process is the fairly noisy nature of the surface brightness profile and/or the optical appearance of the galaxy. Good examples of this are shown in Figure 5 (from O'Neil, et al 1997a, 1997b). P2-4, with $\mu_{B}(0)=25.1 \mathrm{mag} \operatorname{arcsec}^{-2}$ and its highly clumped morphology, is an extreme example of this. It completely lacks any centralized core, and consists primarily of localized regions of above average stellar density. We would identify these as local star forming clumps (the clumps are rather blue). P3-3 is a more common example of a LSB galaxy, with has a definite exponential surface brightness profile and fairly even color profile, but with numerous bumps and wiggles present that are likely manifest of an irregular distribution of 
recent star formation.

For the red LSB galaxies we definitely detect a group with $\mathrm{B}-\mathrm{V}$ and $\mathrm{V}-\mathrm{I}$ colors indicative of an old stellar population but U-B colors indicating current star formation (see O'Neil et al 1997a). If these galaxies had colors similar to those of very red LSB galaxies and then

underwent a burst of star formation, their colors could easily be changed by up to $\Delta(\mathrm{U}-\mathrm{B})$ $=-0.4$ (for a high $\mathrm{M}_{\text {gas }} / \mathrm{L}_{\mathrm{T}}$ ratio), while their $\mathrm{V}-\mathrm{I}$ and $\mu_{B}(0)$ are virtually unchanged. An example of this can be seen in models h4 and h5, where the final galaxy colors are fairly close to the colors of U1-8 in O'Neil et al 1997a $(U-B=0.31, B-I=2.79)$, with final colors of $\mathrm{U}-\mathrm{B}=0.04, \mathrm{~B}-\mathrm{I}=2.3$ and $\mathrm{U}-\mathrm{B}=0.26, \mathrm{~B}-\mathrm{I}=3.3$ for models $\mathrm{h} 4$ and $\mathrm{h} 5$, respectively.

These changes easily explain the colors of U1-8 and similar galaxies, and can account for part of the much low $\mathrm{U}-\mathrm{B}$ colors of $\mathrm{C} 5-3$. It is thus likely these galaxies are older galaxies which are now or have recently experienced a tidal interaction which triggered significant starburst. Most of these galaxies are located in the outskirts of the Pegasus and Cancer clusters of galaxies. Weak/distant tidal encounters would be expected in such an environment.

\section{Conclusion}

We ran 53 different simulations, varying all possible galaxy parameters and allowed up to $75 \%$ of the galaxy's gas to undergo starburst. While we could significantly change the galaxies' total color, creating very blue LSB galaxies, it was virtually impossible (without placing a high threshold criteria for the gas) to significantly alter the galaxy's central surface brightness. Instead the primary effect of large amounts of induced star formation was to produce a centralized core (bulge) component. While some LSBs have this 
component (e.g. Malin 1), most are devoid of any central luminosity excess above the fitted exponential which strongly suggests that such centralized bursts didn't happen in these systems. We also suggest that LSB galaxies evolve through sporadic bursts of star formation and that the colors and noisy morphologies displayed by many detected LSB galaxies can be explained by recent starbursts triggered through distant/weak tidal interaction. Since the observed current SFRs are an order of magnitude too low to produce the observed luminosity in LSB disks, it seems clear that some sort of episodic star formation has occurred.

Our modeling procedure has assumed that $(\delta \rho / \rho)_{\text {gas }} \propto(\delta \rho / \rho)_{\text {stars }}$ in LSB galaxies. De Blok \& McGaugh (1997) have already shown, through analysis of rotation curves, that the low surface light density of these systems does translate into low surface mass density. We believe this to be the basic physical difference between HSB and LSB disks that should directly translate into different star formation histories. Of course, one should question this assumption as it potentially leads to the following dilemma: if $(\delta \rho / \rho)_{\text {gas }} \propto(\delta \rho / \rho)_{\text {stars }}$ in a continuous manner then how come do the very low $\mu_{B}(0)$ systems have any stars in them at all, provided a molecular cloud medium is a prerequisite for large scale star formation to occur in any disk galaxy.? On the other hand if $(\delta \rho / \rho)_{\text {gas }}$ is the similar between high and low surface brightness disks, one is very hard pressed to understand why star formation appears to be so different in the LSBs and/or the lack of dust/heavy elements in LSBs relative to HSBs of the same $V_{c}$.

We suspect that the actual truth lies somewhere between these two extremes but that truth will be elusive. While this is the subject of a larger investigation (O'Neil, Bothun \& Carollo 1998) the executive summary is that, in a LSB disk, to obtain the equivalent gas mass which is, say, contained in a GMC in our Galaxy, requires a significantly larger length scale. When the disk of our model LSB galaxy is broken up into cells, the net result of this 
large length scale requirement is that the probability of massive star formation per cell is low. This predicted dearth of massive star formation, of course, is consistent with the low metallicity and dust content observed in LSB disks and would result in a very slowly evolving population but leaves open the question of how such low surface density disks could have formed in the first place.

To overcome this dearth of massive star formation we have run a set of models that fixes a threshold column density of $\mathrm{H}$ I that remains so even as $\mu_{B_{i}}(0)$ decreases. In this case, there is significantly more gas available for star formation and some of the limitations of the previous models are overcome. However, in most cases the threshold models cause an inner $\mathrm{r}^{1 / 4}$ component to develop. Thus the LSB disk gains a "bulge" in response to the starburst and this is generally not observed. To effect a large increase in $\mu_{B}(0)$ with a localized disk starburst event generally requires an inordinate amount of gas to be converted into stars. Moreover, its unclear that the threshold model is able to preserve the observed and important non-correlation between $\mu_{B}(0)$ and disk color or account for the offset in mean $\log \mathrm{O} / \mathrm{H}$ at a given $V_{c}$ with respect to $\mathrm{HSB}$ galaxies. Most worrisome about the threshold model is its implication that LSB disks of arbitrarily low $\mu_{B}(0)$ are sitting around with ample amounts of gas. To date, systems like this have not been discovered in blind H I surveys. Hence we believe that the slow evolutionary rate of LSB disks is likely controlled by low gas density that accompanies low $\mu_{B}(0)$ which precludes the formation of very many massive stars per star formation event.

Our primary result from the modeling procedure is that once $\mu_{B}(0)$ is established for LSB systems, it is extremely difficult to alter it. That is, disk systems are quite unlikely to hop back and forth between states of high and low surface brightness due to episodic star formation. We thus conclude that if a galaxy forms as a LSB galaxy, due to low gas density, environmental conditions, etc, it will remain a LSB galaxy barring any major 
encounter catastrophe. This implies that the large space density of LSB galaxies at $\mathrm{z}=0$ should hold to substantially higher redshifts. This may have relevance to understanding the nature of QSO absorption line systems at these redshifts (see Linder 1998). This also suggests that deep CCD surveys should reveal this population, if those surveys are relatively free of selection effects. This selection effects, of course, will be more severe than those associated with finding $z=0$ LSB systems due to the significant $(1+z)^{4}$ dilution factor. 


\section{References}

Alvensleben, F-V. 1998, preprint

Bahcall, J., Hut, P., \& Tremaine, S. 1985, ApJ, 290, 15

Becker, S. 1981, ApJS, 45, 475

Becker, S. \& Iben, I. 1979, ApJ, 232, 831

Bothun, G.D. \& McGaugh, S 1998 in preparation

Bothun, G.D., Impey, C., \& McGaugh, S. 1997 PASP 109, 745

Bothun, Gregory D., Schombert, James M., Impey, Christopher D., Sprayberry, David, \&

McGaugh, Stacy S. 1993 AJ 106, 530

Bothun, G.D., et al 1990 ApJ, 360, 427

Bothun, Gregory D., Mould, Jeremy R., Caldwell, Nelson, \& MacGillivray, Harvey T. 1986

AJ 92, 1007

Bothun, G.D., \& Schommer, R.A. 1982 AJ 87, 1368

Briggs, F.H. 1990 AJ, 100, 999

Bushouse, H. 1986, AJ, 91, 255

Caldwell, N., \& Bothun, G.D. 1987 AJ 94, 1126

Davies, R. L., Bertschinger, E., \& Baggley, G. 1993 MNRAS 262, 475

Dalcanton, Julianne J., Spergel, David N., Gunn, James E., Schmidt, Maarten, \&

Schneider, Donald P. 1997 AJ 114, 63

De Blok, W. 1997, Ph.D. thesis University of Groningen, Groningen, Netherlands

De Blok, W., \& McGaugh, S. 1997, MNRAS 290, 533

De Blok, W., Bothun, G., \& van der Hulst, J. 1995, MNRAS, 274, 23

De Jong, R.S. 1996 A\&A, 313, 377

Downes, \& Solomon, 1998 ApJ, in press

Garnett, D. R., et al 1997 ApJ, 489, 63

Guiderdoni, B. \& Rocca-Volmerange, B. 1987, A\&A, 186, 1 
Han, et al 1998 ApJ, in press

Heckman, T. M., et al 1998 preprint

Ichikawa, S. -I., Wakamatsu, K. -I., \& Okamura, S. 1986 ApJS 60, 475

Impey, C. \& Bothun, G. 1997, ARA\&A 35, 267

Impey, C. Sprayberry, D.. Irwin, M. J., \& Bothun, G. D. 1996, ApJS, 105, 209

Impey, C. \& Bothun, G. 1989, ApJ 341, 89

Kennicutt, R. 1989, ApJ 344, 685

Kennicutt, R., et al 1987, AJ, 93, 1011

Knezeck, P., 1993 Ph.D. Thesis University of Pennsylvania

Larson, R., \& Tinsley, B. 1978, AJ, 219, 46

Linder, Suzanne 19981998 ApJ 495, 637

McGaugh, S., Bothun, G., \& Schombert, J. 1995, AJ 110, 573

McGaugh, S. 1994, ApJ 426, 135

McGaugh, S., \& Bothun, G. 1994, AJ, 107, 530

McGaugh, S., \& de Blok, W. 1993, AJ 106, 548

McGaugh, S. 1992, Ph.D. thesis, University of Michigan, Ann Arbor

Mengel, J., et al 1979, ApJS, 40, 733

Mihos, C., \& Bothun, G. 1998 ApJ, in press

Mihos, C., de Block, W., \& McGaugh, S. 1997, ApJ, 477, L79

Mihos, C. \& Hernquist, L. 1996, ApJ, 464, 641

Mihos, C. \& Hernquist, L. 1994, ApJ, 425, L13

Mihos, C., Bothun, G., \& Richstone, D. 1993, ApJ, 418, 82

Mihos, C., Richstone, D., \& Bothun, G. 1991, ApJ, 377, 72

Mo, H. J., McGaugh, Stacy S., Bothun, Gregory D. 1994 MNRAS 267, 129

Oey, M.S. \& Clarke, C.J. 1998 AJ, 115, 1543

Oey, M.S. \& Kennicutt, R.C. Jr. 1997 MNRAS, 289, 570 
O’Neil, K., 1998 in preparation

O’Neil, K., Carollo, \& Bothun, G.D. 1998, in preparation

O’Neil, K., Bothun, G., Impey, C., McGaugh, S. 1998a, AJ, 119

O’Neil, K., Bothun, G., \& Impey, C. 1998b, AJ, submitted

O’Neil, K. 1997, Ph.D. thesis, University of Oregon, Eugene

O’Neil, K., Bothun, G., Schombert, J., Cornell, M., \& Impey, C. 1997a, AJ 114, 2448

O’Neil, K., Bothun, G., \& Cornell, M. 1997b, AJ 113, 1212

Pickering, T., Impey, C., Van Gorkom, J, Bothun, G. 1997, AJ, 114, 1858

Schaller, G., et al 1992, A \& AS, 96, 269

Schombert, James M., Bothun, Gregory D., Schneider, Stephen E., \& McGaugh, Stacy S. 1992 AJ, 103, 1107

Salzer, J. 1998, preprint

Schombert J., et al 1990, AJ, 100, 1523

Skillman, E. D., 1998, preprint

Skillman, E. D., Bothun, G. D., Murray, M. A., \& Warmels, R. H. 1987 A\&A, 185, 61

Smith, Denise A., et al 1996 ApJ 473L, 21

Spitzak, \& Schneider 1998, preprint

Sprayberry, D., Impey, C. D., Irwin, M. J., \& Bothun, G. D. 1997a ApJ 482, 104

Sprayberry, D., Bernstein, G.M., Impey, C. D., \& Bothun, G. D. 1997b ApJ 438, 72

Sprayberry, D., Impey, C. D., Bothun, G. D., Irwin, M. J. 1995 AJ 109, 558

Sprayberry, D., Impey, C. D., Bothun, G. D., \& Irwin, M. J. 1995 AJ, 109, 558

Sweigart, A. \& Gross, P. 1978, ApJS, 36, 405

Van Der Hulst, J. M., Skillman, E. D., Smith, T. R., Bothun, G. D., McGaugh, S. S., \& de

Blok, W. J. G. 1993 AJ 106, 548

Van Zee, Liese, Haynes, Martha P., Salzer, John J., \& Broeils, Adrick H. 1997 AJ, 113, 1618

Vázquez, E. \& Scalo, J. 1989, ApJ, 343, 644 
Webster, R., et al 1998, preprint 
Figure 1. The before and after gas density for various models. Figure 1(a) shows model a2 before (solid line) and after (dashed line) the starburst as well as model 12 (dash-dotted line before and dotted line after). Figure 1 (b) shows models a3 (solid line before and dashed line after) and 13 (dash-dotted line before and dotted line after). Finally, Figure 1 1 (c) shows models i3 (solid line before and dashed line after) and 14 (dash-dotted line before and dotted line after).

Figure 2. The starburst distribution for four of the models - c2, c5, f1, and $\mathrm{f} 2$. The shading is proportional to the total mass involved in the starburst. Thus the darkest regions on the plot typically lie at the core of the starburst while the white regions were unaffected by the starburst (no gas was converted to stars). Models c2 and c5 show the effects of moving the burst radius from $0.10 \mathrm{R}_{T}$ to $0.80 \mathrm{R}_{T}$, while models $\mathrm{f} 1$ and $\mathrm{f} 2$ show the effects of altering the concentration parameter c from 1.0 to 5.0 .

Figure 3. The distribution of stellar masses for four different models - model e2 (solid line), model e1 (dashed line), model e3 (dash-dotted line), and model j5 (dotted line). Model e2 (solid line) is representative of the majority of the models.

Figure 4 . The color and surface brightness plots for each of the models. The top plot is the change in total $\mathrm{U}-\mathrm{B}$ color, with time, for each of the models. The bottom two plots show the surface brightness profile and $\mathrm{U}-\mathrm{B}$ color profile of each model 2 Myr after the burst.

Figure 5. Examples of surface brightness and color profiles of two LSB galaxies - P2-4 $\left(\mu(0)=25.14 \mathrm{mag} \operatorname{arcsec}^{-2}\right.$, Figure 5(a) \& (b)), and P3-3 $\left(\mu(0)=23.22 \mathrm{mag} \operatorname{arcsec}^{-2}\right.$, Figure 5(c) \& (d)) (from O’Neil, et al , 1997a, 1997b). 
Table 1. Results for the one cell models discussed in section 3.1 (Table 2). The numbers given are the change in total color and magnitude between $\mathrm{t}=0$ (before the starburst) and $\mathrm{t}=2 \mathrm{Myr}$.

Table 2. The model parameters for all models discussed in this paper.

Table 3. Results for the models discussed in this paper (Table 2). The numbers given are the change in total color and magnitude between $t=0$ (before the starburst) and $t=2 \mathrm{Myr}$.

Table 4 . Comparison of the results for models a1-a3 \& i3 with models 11-14. The numbers given are the change in total color and magnitude between $\mathrm{t}=0$ (before the starburst) and $\mathrm{t}=2 \mathrm{Myr}$. 
Table 1:

Table 2:

Table 3:

Table 4: 
Fig. 1.-

Fig. 2.-

Fig. 3.- 
Fig. 4.-

Fig. 5.- a

Fig. 5.- b

Fig. 5.- c

Fig. 5.- d 


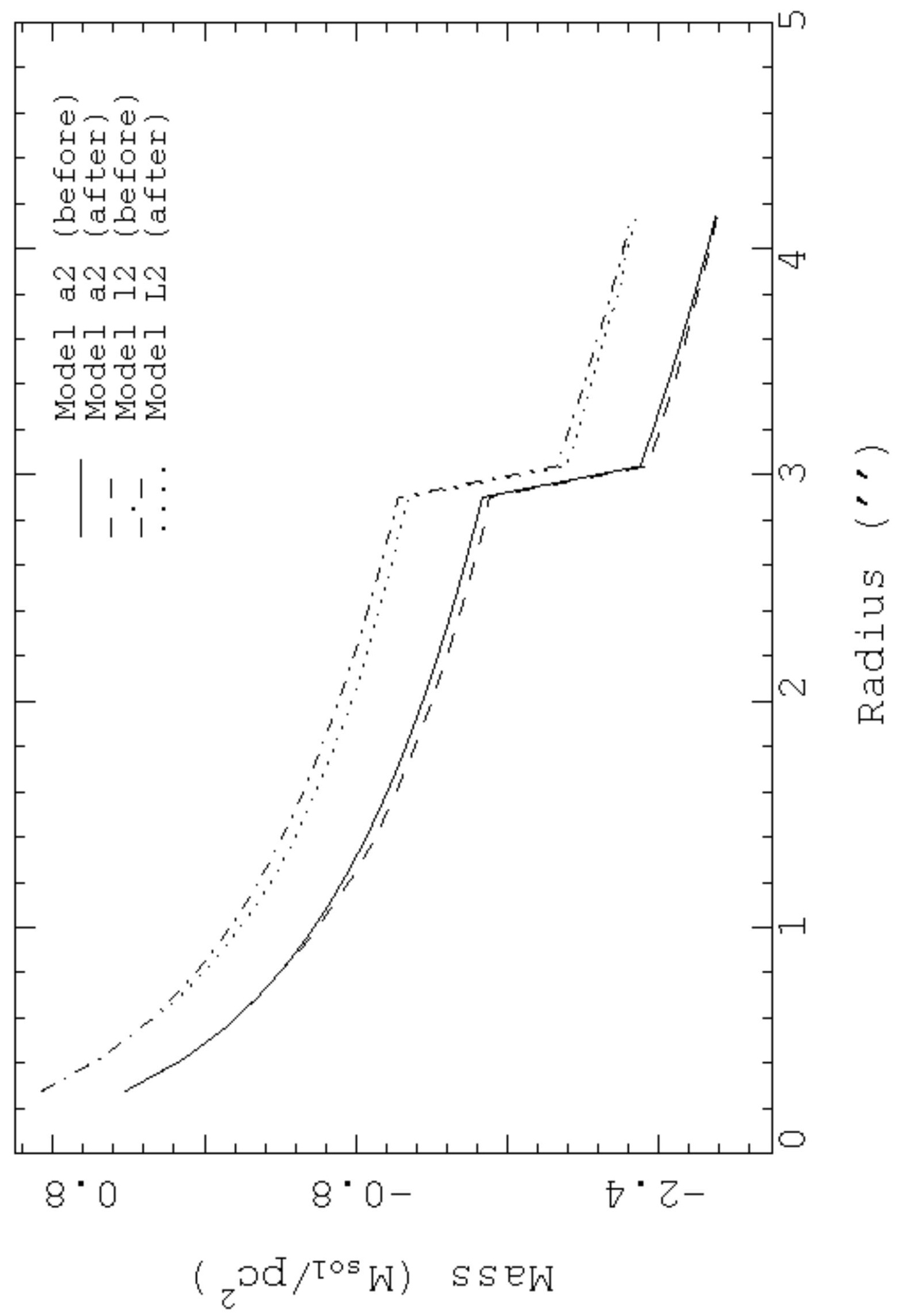




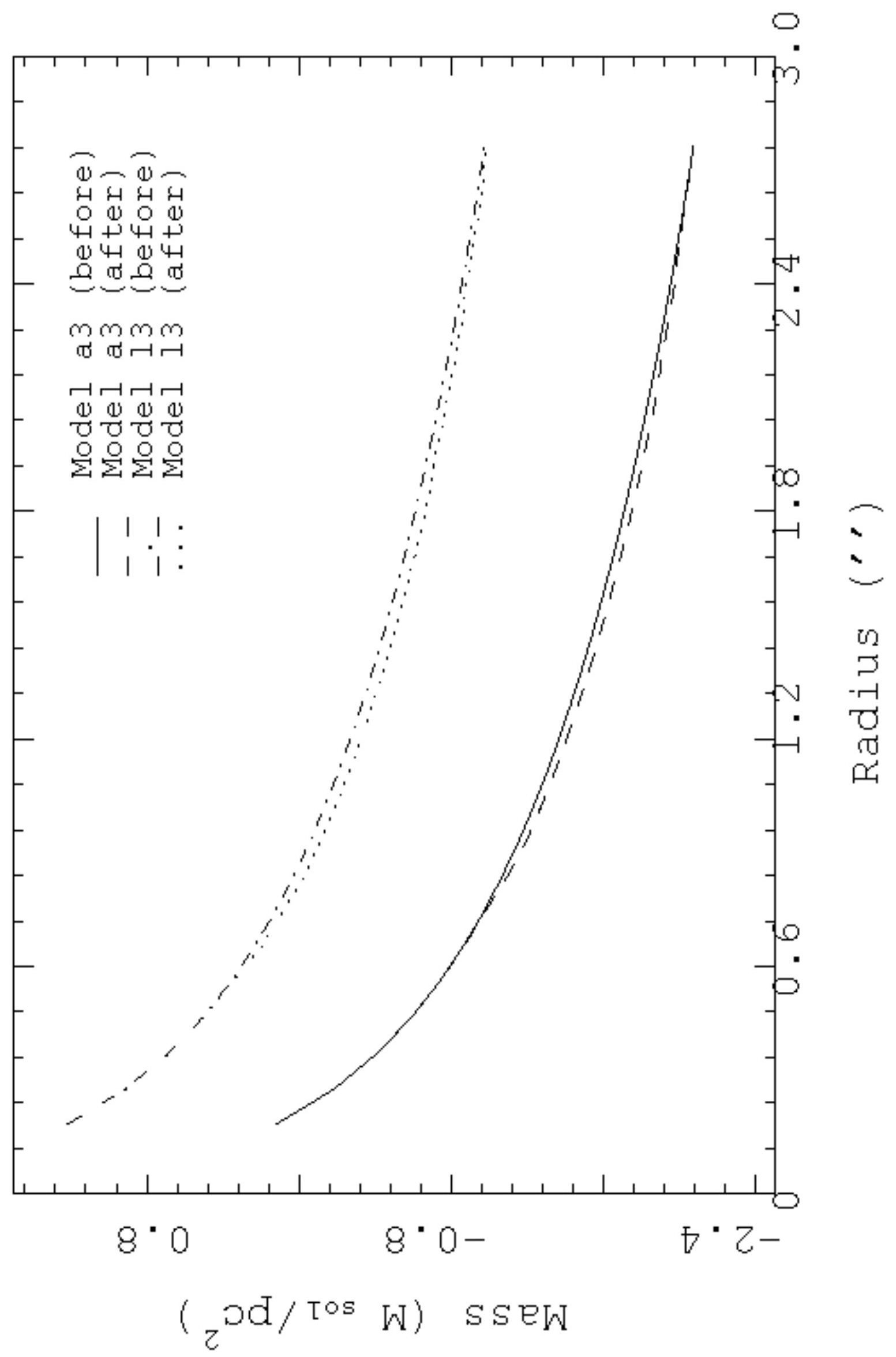




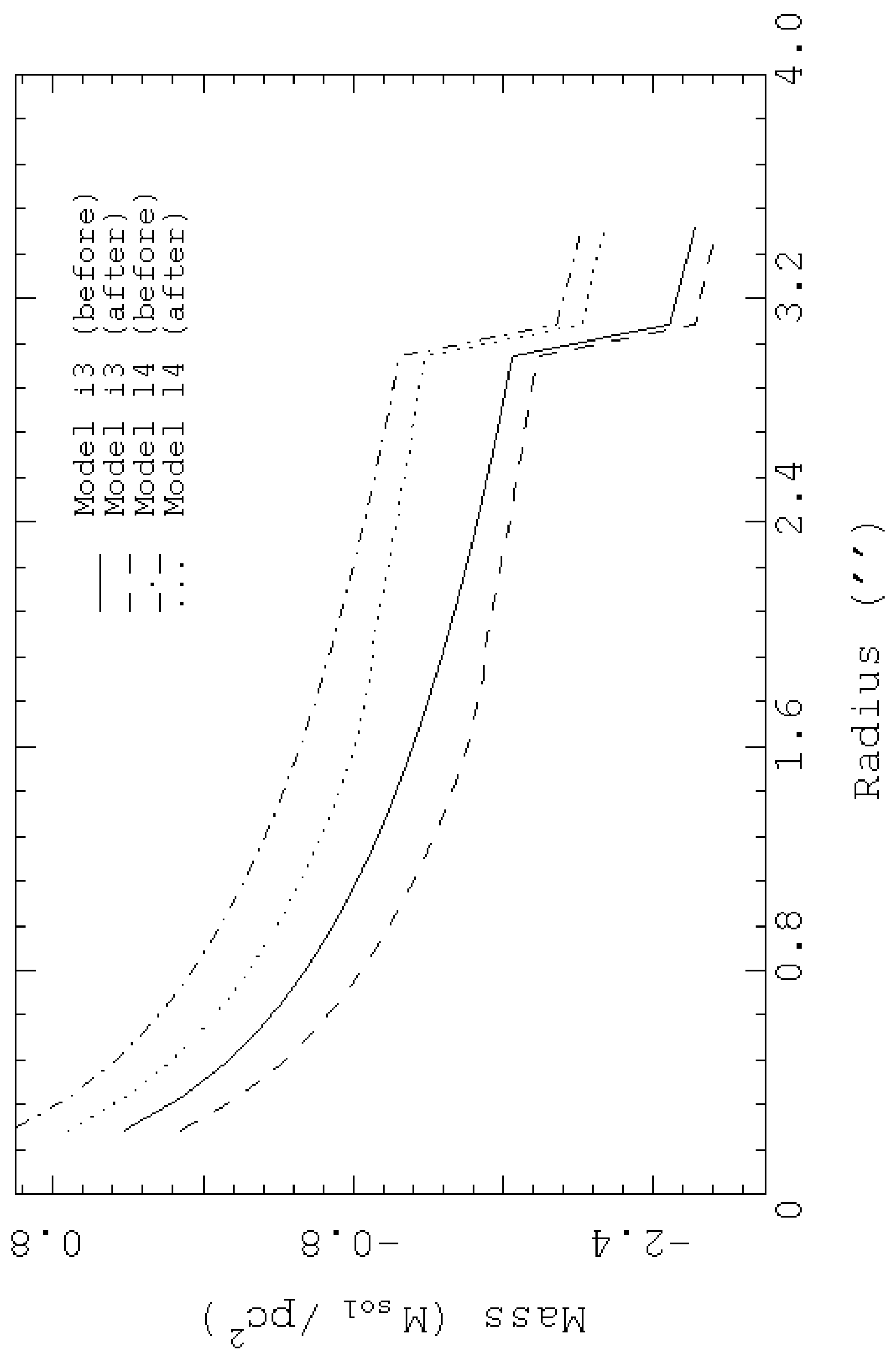


Table 3:

\begin{tabular}{|c|c|c|c|c|c|c|c|c|c|c|c|}
\hline Model & $\Delta(\mathrm{U}-\mathrm{B})$ & $\Delta(\mathrm{B}-\mathrm{V})$ & $\Delta(\mathrm{V}-\mathrm{I})$ & $\Delta \mathrm{B}_{\mathrm{mag}}$ & $\frac{\Delta \mathrm{L}}{\mathrm{L}}$ & Model & $\Delta(\mathrm{U}-\mathrm{B})$ & $\Delta(\mathrm{B}-\mathrm{V})$ & $\Delta(\mathrm{V}-\mathrm{I})$ & $\Delta \mathrm{B}_{\mathrm{mag}}$ & $\frac{\Delta \mathbf{L}}{\mathbf{L}}$ \\
\hline a1 & -0.33 & -0.17 & -0.09 & -0.30 & 0.32 & g1 & -0.20 & -0.09 & -0.05 & -0.15 & 0.16 \\
\hline $\mathrm{a} 2$ & -0.33 & -0.17 & -0.09 & -0.30 & 0.32 & g2 & -0.33 & -0.17 & -0.09 & -0.29 & 0.31 \\
\hline a3 & -0.19 & -0.09 & -0.04 & -0.15 & 0.15 & g3 & -0.42 & -0.24 & -0.13 & -0.42 & 0.48 \\
\hline b1 & -0.32 & -0.17 & -0.09 & -0.29 & 0.31 & g4 & -0.49 & -0.29 & -0.17 & -0.49 & 0.63 \\
\hline $\mathrm{b} 2$ & -0.33 & -0.17 & -0.09 & -0.30 & 0.32 & g5 & -0.55 & -0.34 & -0.20 & -0.63 & 0.79 \\
\hline b3 & -0.33 & -0.17 & -0.09 & -0.30 & 0.32 & h1 & -0.23 & -0.17 & -0.14 & -0.41 & 0.48 \\
\hline $\mathrm{b} 4$ & -0.29 & -0.15 & -0.07 & -0.25 & 0.26 & h2 & -0.32 & -0.21 & -0.14 & -0.41 & 0.48 \\
\hline c1 & -0.42 & -0.24 & -0.13 & -0.42 & 0.48 & h3 & -0.42 & -0.24 & -0.13 & -0.41 & 0.48 \\
\hline$c 2$ & -0.42 & -0.24 & -0.13 & -0.42 & 0.48 & h4 & -0.54 & -0.27 & -0.12 & -0.41 & 0.48 \\
\hline c3 & -0.42 & -0.24 & -0.13 & -0.42 & 0.48 & h5 & -0.66 & -0.32 & -0.09 & -0.41 & 0.48 \\
\hline c4 & -0.42 & -0.24 & -0.13 & -0.42 & 0.48 & i1 & -0.55 & -0.34 & -0.20 & -0.63 & 0.79 \\
\hline$c 5$ & -0.42 & -0.24 & -0.13 & -0.42 & 0.48 & $i 2$ & -0.70 & -0.51 & -0.35 & -1.04 & 1.60 \\
\hline $\mathrm{d} 1$ & -0.06 & -0.03 & -0.01 & -0.04 & 0.04 & i3 & -0.78 & -0.62 & -0.46 & -1.32 & 2.40 \\
\hline $\mathrm{d} 2$ & -0.27 & -0.13 & -0.07 & -0.22 & 0.23 & i4 & -0.91 & -0.85 & -0.85 & -2.38 & 7.99 \\
\hline $\mathrm{d} 3$ & -0.42 & -0.24 & -0.13 & -0.42 & 0.48 & 15 & -0.95 & -0.93 & -1.04 & -3.07 & 16.0 \\
\hline $\mathrm{d} 4$ & -0.59 & -0.39 & -0.23 & -0.72 & 0.96 & & & & & & \\
\hline $\mathrm{d} 5$ & -0.68 & -0.49 & -0.32 & -0.96 & 1.43 & & & & & & \\
\hline e1 & -0.12 & -0.06 & -0.03 & -0.09 & 0.09 & & & & & & \\
\hline $\mathrm{e} 2$ & -0.42 & -0.24 & -0.13 & -0.42 & 0.48 & & & & & & \\
\hline e3 & -0.59 & -0.39 & -0.23 & -0.72 & 0.96 & & & & & & \\
\hline f1 & -0.42 & -0.24 & -0.13 & -0.42 & 0.48 & & & & & & \\
\hline f2 & -0.42 & -0.24 & -0.13 & -0.42 & 0.48 & & & & & & \\
\hline f3 & -0.42 & -0.24 & -0.13 & -0.42 & 0.48 & & & & & & \\
\hline f4 & -0.42 & -0.24 & -0.13 & -0.42 & 0.48 & & & & & & \\
\hline f5 & -0.42 & -0.24 & -0.13 & -0.42 & 0.48 & & & & & & \\
\hline
\end{tabular}



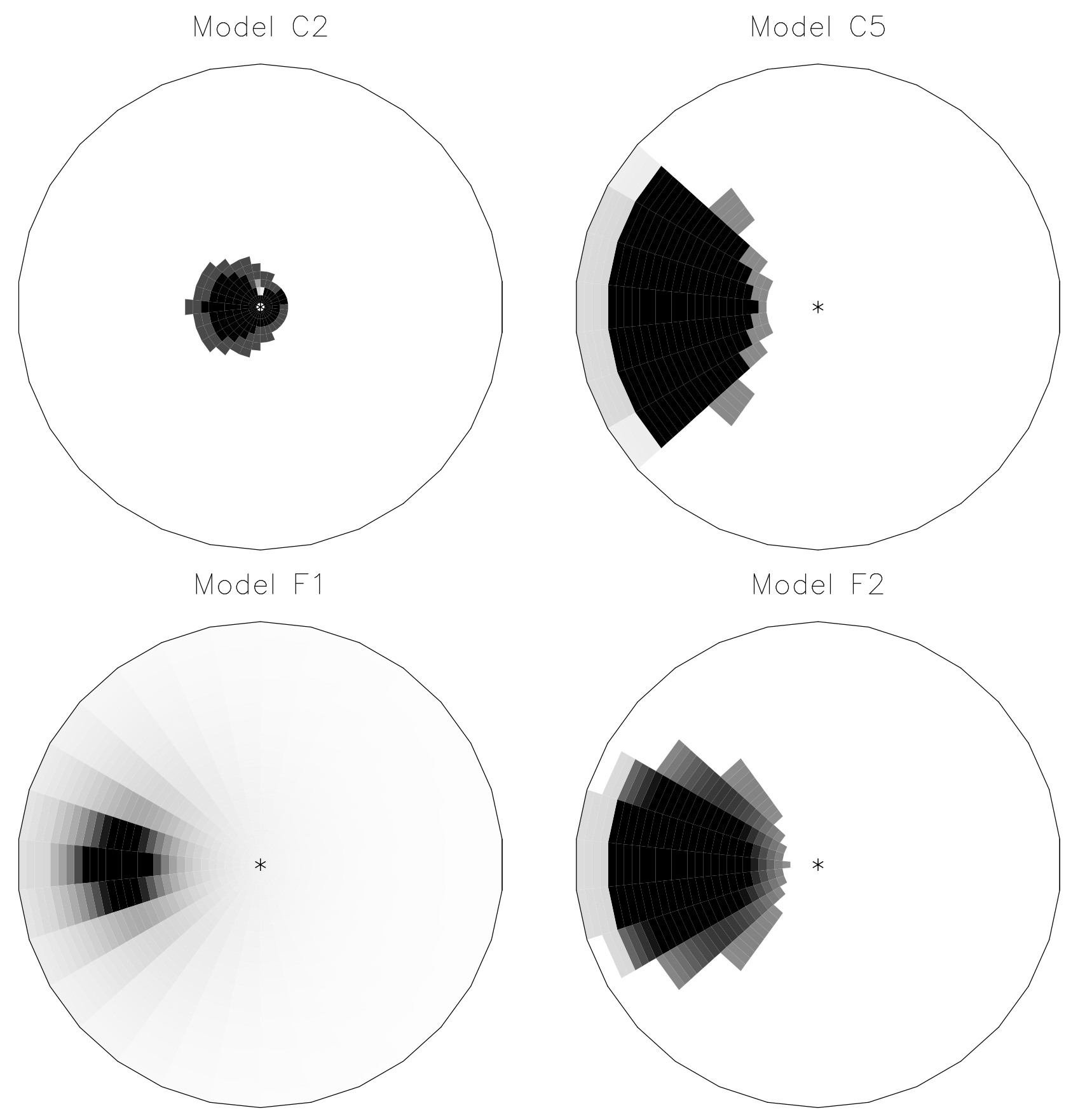


\begin{tabular}{|c|c|c|c|c|c|c|c|c|c|c|}
\hline Model & $\mu(0)$ & $\alpha(\mathrm{kpc})$ & $\mathrm{r}_{\text {burst }} / \mathrm{r}_{\mathrm{T}}$ & $\mathbf{M}_{\mathbf{T}} / \mathbf{L}_{\mathbf{T}}$ & $\mathrm{M}_{\mathrm{gas}} / \mathrm{M}_{\mathrm{T}}$ & c & $\frac{m_{\text {burst }}}{m_{\text {gas }}}$ & U-B & $B-V$ & V-I \\
\hline a1 & 23.0 & 1.5 & 0.60 & 10.0 & 0.05 & 40.0 & 0.10 & 0.20 & 0.80 & 1.20 \\
\hline $\mathrm{a} 2$ & 24.0 & 1.5 & 0.60 & 10.0 & 0.05 & 40.0 & 0.10 & 0.20 & 0.80 & 1.20 \\
\hline $\mathrm{a} 3$ & 25.0 & 1.5 & 0.60 & 5.0 & 0.05 & 40.0 & 0.10 & 0.20 & 0.80 & 1.20 \\
\hline b1 & 24.5 & 0.8 & 0.60 & 10.0 & 0.05 & 40.0 & 0.10 & 0.20 & 0.80 & 1.20 \\
\hline $\mathrm{b} 2$ & 24.5 & 2.0 & 0.60 & 10.0 & 0.05 & 40.0 & 0.10 & 0.20 & 0.80 & 1.20 \\
\hline b3 & 24.5 & 4.0 & 0.60 & 10.0 & 0.05 & 40.0 & 0.10 & 0.20 & 0.80 & 1.20 \\
\hline $\mathrm{b} 4$ & 24.5 & 10.0 & 0.60 & 10.0 & 0.05 & 65.0 & 0.10 & 0.20 & 0.80 & 1.20 \\
\hline c1 & 24.5 & 1.5 & even & 10.0 & 0.05 & 20.0 & 0.15 & 0.20 & 0.80 & 1.20 \\
\hline$c 2$ & 24.5 & 1.5 & 0.10 & 10.0 & 0.05 & 20.0 & 0.15 & 0.20 & 0.80 & 1.20 \\
\hline c3 & 24.5 & 1.5 & 0.30 & 10.0 & 0.05 & 20.0 & 0.15 & 0.20 & 0.80 & 1.20 \\
\hline$c 4$ & 24.5 & 1.5 & 0.50 & 10.0 & 0.05 & 20.0 & 0.15 & 0.20 & 0.80 & 1.20 \\
\hline$c 5$ & 24.5 & 1.5 & 0.80 & 10.0 & 0.05 & 20.0 & 0.15 & 0.20 & 0.80 & 1.20 \\
\hline $\mathrm{d} 1$ & 24.5 & 1.5 & 0.60 & 1.0 & 0.05 & 20.0 & 0.15 & 0.20 & 0.80 & 1.20 \\
\hline $\mathrm{d} 2$ & 24.5 & 1.5 & 0.60 & 5.0 & 0.05 & 20.0 & 0.15 & 0.20 & 0.80 & 1.20 \\
\hline $\mathrm{d} 3$ & 24.5 & 1.5 & 0.60 & 10.0 & 0.05 & 20.0 & 0.15 & 0.20 & 0.80 & 1.20 \\
\hline $\mathrm{d} 4$ & 24.5 & 1.5 & 0.60 & 20.0 & 0.05 & 20.0 & 0.15 & 0.20 & 0.80 & 1.20 \\
\hline $\mathrm{d} 5$ & 24.5 & 1.5 & 0.60 & 30.0 & 0.05 & 20.0 & 0.15 & 0.20 & 0.80 & 1.20 \\
\hline e1 & 24.5 & 1.5 & 0.60 & 10.0 & 0.01 & 20.0 & 0.15 & 0.20 & 0.80 & 1.20 \\
\hline $\mathrm{e} 2$ & 24.5 & 1.5 & 0.60 & 10.0 & 0.05 & 20.0 & 0.15 & 0.20 & 0.80 & 1.20 \\
\hline $\mathrm{e} 3$ & 24.5 & 1.5 & 0.60 & 10.0 & 0.10 & 20.0 & 0.15 & 0.20 & 0.80 & 1.20 \\
\hline f1 & 24.5 & 1.5 & 0.60 & 10.0 & 0.05 & 1.0 & 0.15 & 0.20 & 0.80 & 1.20 \\
\hline$f 2$ & 24.5 & 1.5 & 0.60 & 10.0 & 0.05 & 5.0 & 0.15 & 0.20 & 0.80 & 1.20 \\
\hline f3 & 24.5 & 1.5 & 0.60 & 10.0 & 0.05 & 10.0 & 0.15 & 0.20 & 0.80 & 1.20 \\
\hline f4 & 24.5 & 1.5 & 0.60 & 10.0 & 0.05 & 50.0 & 0.15 & 0.20 & 0.80 & 1.20 \\
\hline f5 & 24.5 & 1.5 & 0.60 & 10.0 & 0.05 & 100.0 & 0.15 & 0.20 & 0.80 & 1.20 \\
\hline g1 & 24.5 & 1.5 & 0.60 & 10.0 & 0.05 & 20.0 & 0.05 & 0.20 & 0.80 & 1.20 \\
\hline g2 & 24.5 & 1.5 & 0.60 & 10.0 & 0.05 & 20.0 & 0.10 & 0.20 & 0.80 & 1.20 \\
\hline g3 & 24.5 & 1.5 & 0.60 & 10.0 & 0.05 & 20.0 & 0.15 & 0.20 & 0.80 & 1.20 \\
\hline g4 & 24.5 & 1.5 & 0.60 & 10.0 & 0.05 & 20.0 & 0.20 & 0.20 & 0.80 & 1.20 \\
\hline g5 & 24.5 & 1.5 & 0.60 & 10.0 & 0.05 & 20.0 & 0.25 & 0.20 & 0.80 & 1.20 \\
\hline h1 & 24.5 & 1.5 & 0.60 & 10.0 & 0.05 & 20.0 & 0.15 & -0.20 & 0.40 & 0.80 \\
\hline h2 & 24.5 & 1.5 & 0.60 & 10.0 & 0.05 & 20.0 & 0.15 & 0.00 & 0.60 & 1.00 \\
\hline h3 & 24.5 & 1.5 & 0.60 & 10.0 & 0.05 & 20.0 & 0.15 & 0.20 & 0.80 & 1.20 \\
\hline h4 & 24.5 & 1.5 & 0.60 & 10.0 & 0.05 & 20.0 & 0.15 & 0.40 & 1.00 & 1.40 \\
\hline h5 & 24.5 & 1.5 & 0.60 & 10.0 & 0.05 & 20.0 & 0.15 & 0.60 & 1.50 & 2.00 \\
\hline i1 & 24.5 & 1.5 & 0.60 & 10.0 & 0.05 & 50.0 & 0.25 & 0.20 & 0.80 & 1.20 \\
\hline$i 2$ & 24.5 & 1.5 & 0.60 & 10.0 & 0.05 & 50.0 & 0.50 & 0.20 & 0.80 & 1.20 \\
\hline i3 & 24.5 & 1.5 & 0.60 & 10.0 & 0.05 & 50.0 & 0.75 & 0.20 & 0.80 & 1.20 \\
\hline i4 & 24.5 & 1.5 & 0.60 & 10.0 & 0.25 & 50.0 & 0.50 & 0.20 & 0.80 & 1.20 \\
\hline i. 5 & 24.5 & 1.5 & 0.60 & 10.0 & 0.50 & 50.0 & 0.50 & 0.20 & 0.80 & 1.20 \\
\hline j1 & 24.5 & 1.5 & 0.60 & 10.0 & 0.05 & 20.0 & 0.15 & 0.20 & 0.80 & 1.20 \\
\hline j2 & 24.5 & 1.5 & 0.60 & 10.0 & 0.15 & 20.0 & 0.15 & 0.20 & 0.80 & 1.20 \\
\hline j3 & 24.5 & 1.5 & 0.10 & 10.0 & 0.15 & 20.0 & 0.15 & 0.20 & 0.80 & 1.20 \\
\hline $\mathrm{j} 4$ & 24.5 & 1.5 & even & 10.0 & 0.05 & 20.0 & 0.15 & 0.20 & 0.80 & 1.20 \\
\hline $\mathrm{j} 5$ & 24.5 & 1.5 & even & 10.0 & 0.15 & 20.0 & 0.15 & 0.20 & 0.80 & 1.20 \\
\hline $\mathrm{k} 1$ & 24.5 & 1.5 & even & 10.0 & 0.05 & 50.0 & 0.25 & 0.20 & 0.80 & 1.20 \\
\hline $\mathrm{k} 2$ & 24.5 & 1.5 & even & 10.0 & 0.05 & 50.0 & 0.50 & 0.20 & 0.80 & 1.20 \\
\hline $\mathrm{k} 3$ & 24.5 & 1.5 & even & 10.0 & 0.25 & 50.0 & 0.50 & 0.20 & 0.80 & 1.20 \\
\hline $\mathrm{k} 4$ & 24.5 & 1.5 & even & 10.0 & 0.50 & 50.0 & 0.50 & 0.20 & 0.80 & 1.20 \\
\hline 11 & 23.0 & 1.5 & 0.60 & 10.0 & 0.05 & 20.0 & 0.15 & 0.20 & 0.80 & 1.20 \\
\hline 12 & 24.0 & 1.5 & 0.60 & 10.0 & 0.05 & 20.0 & 0.15 & 0.20 & 0.80 & 1.20 \\
\hline 13 & 25.0 & 1.5 & 0.60 & 10.0 & 0.05 & 20.0 & 0.15 & 0.20 & 0.80 & 1.20 \\
\hline 14 & 24.5 & 1.5 & 0.60 & 10.0 & 0.05 & 50.0 & 0.75 & 0.20 & 0.80 & 1.20 \\
\hline
\end{tabular}




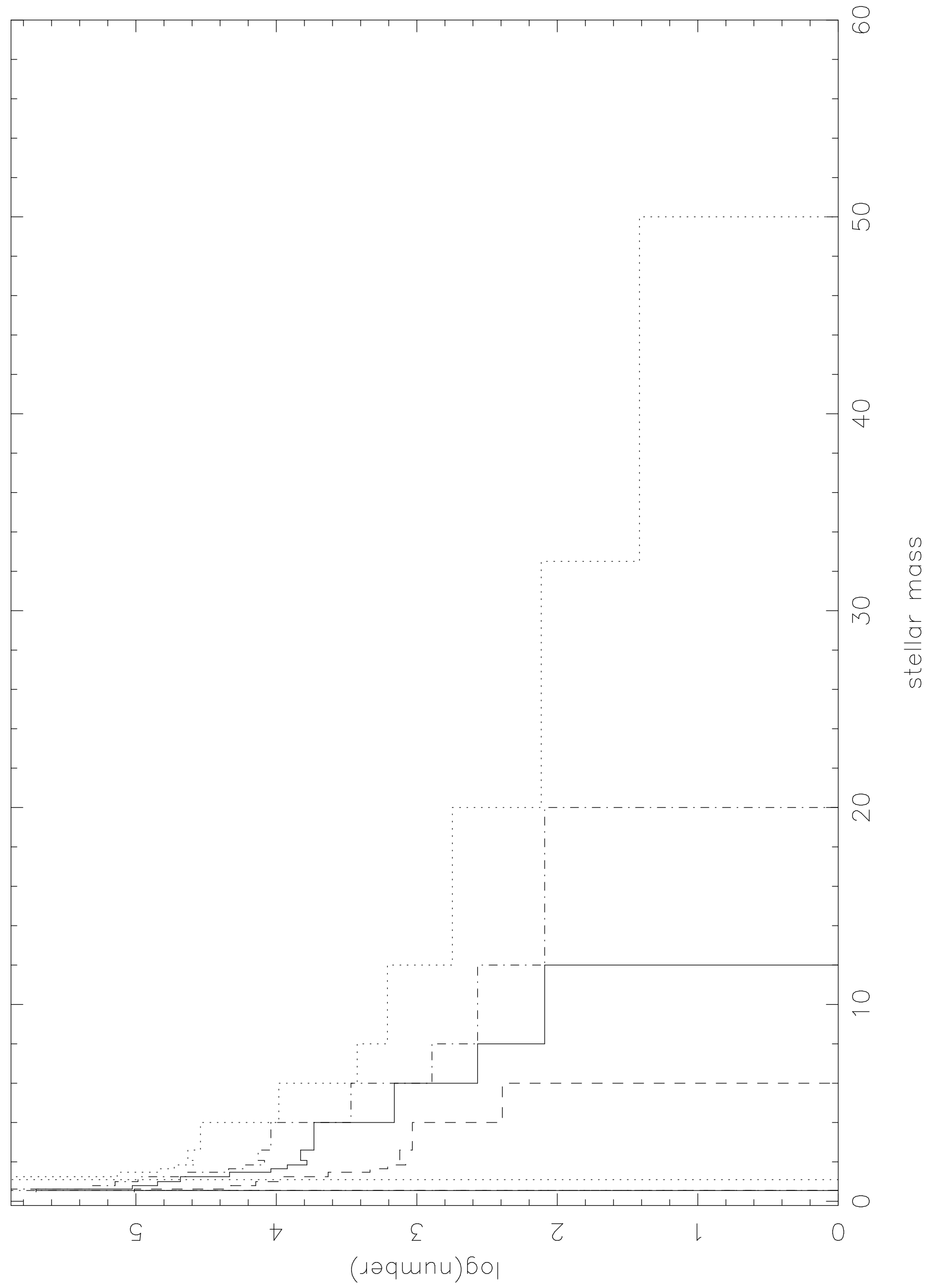


Table 2:

\begin{tabular}{|c|c|c|c|c|c|c|c|c|c|c|c|}
\hline Model & $\Delta(\mathrm{U}-\mathrm{B})$ & $\Delta(\mathrm{B}-\mathrm{V})$ & $\Delta(\mathrm{V}-\mathrm{I})$ & $\Delta B_{\text {mag }}$ & $\frac{\Delta \mathrm{L}}{\mathrm{L}}$ & Model & $\Delta(\mathrm{U}-\mathrm{B})$ & $\Delta(\mathrm{B}-\mathrm{V})$ & $\Delta(\mathrm{V}-\mathrm{I})$ & $\Delta B_{\mathrm{mag}}$ & $\frac{\Delta \mathrm{L}}{\mathrm{L}}$ \\
\hline a1 & -0.16 & -0.10 & -0.05 & -0.18 & 0.17 & g1 & -0.05 & -0.03 & -0.01 & -0.07 & 0.08 \\
\hline $\mathrm{a} 2$ & -0.17 & -0.10 & -0.05 & -0.17 & 0.17 & g2 & -0.13 & -0.07 & -0.04 & -0.13 & 0.13 \\
\hline a3 & -0.04 & -0.03 & -0.01 & -0.05 & 0.04 & g3 & -0.19 & -0.12 & -0.06 & -0.21 & 0.22 \\
\hline b1 & -0.07 & -0.02 & -0.05 & -0.02 & 0.09 & g4 & -0.25 & -0.16 & -0.09 & -0.28 & 0.30 \\
\hline $\mathrm{b} 2$ & -0.15 & -0.09 & -0.04 & -0.14 & 0.14 & g5 & -0.28 & -0.18 & -0.10 & -0.33 & 0.36 \\
\hline b3 & -0.14 & -0.07 & -0.03 & -0.20 & 0.21 & h1 & -0.08 & -0.08 & -0.07 & -0.21 & 0.22 \\
\hline b4 & -0.21 & -0.11 & -0.06 & -0.20 & 0.20 & h2 & -0.14 & -0.10 & -0.07 & -0.21 & 0.22 \\
\hline $\mathrm{c} 1$ & -0.27 & -0.17 & -0.09 & -0.29 & 0.32 & h3 & -0.20 & -0.12 & -0.06 & -0.21 & 0.22 \\
\hline$c 2$ & -0.24 & -0.15 & -0.08 & -0.25 & 0.27 & $\mathrm{~h} 4$ & -0.26 & -0.13 & -0.06 & -0.21 & 0.22 \\
\hline$c 3$ & -0.19 & -0.11 & -0.06 & -0.19 & 0.20 & h. 5 & -0.34 & -0.16 & -0.04 & -0.21 & 0.22 \\
\hline$c 4$ & -0.20 & -0.12 & -0.07 & -0.21 & 0.22 & i1 & -0.28 & -0.18 & -0.10 & -0.33 & 0.36 \\
\hline$c 5$ & -0.23 & -0.14 & -0.08 & -0.25 & 0.26 & $i 2$ & -0.48 & -0.36 & -0.23 & -0.70 & 0.91 \\
\hline $\mathrm{d} 1$ & -0.02 & -0.01 & -0.01 & -0.01 & 0.02 & i3 & -0.56 & -0.47 & -0.32 & -0.97 & 1.34 \\
\hline $\mathrm{d} 2$ & -0.08 & -0.05 & -0.03 & -0.09 & 0.09 & i4 & -0.73 & -0.73 & -0.66 & -1.90 & 4.76 \\
\hline $\mathrm{d} 3$ & -0.20 & -0.12 & -0.06 & -0.21 & 0.22 & 15 & -0.86 & -0.88 & -0.94 & -2.74 & 11.5 \\
\hline $\mathrm{d} 4$ & -0.34 & -0.23 & -0.13 & -0.41 & 0.47 & j1 & -0.31 & -0.22 & -0.12 & -0.39 & 0.44 \\
\hline $\mathrm{d} 5$ & -0.32 & -0.20 & -0.11 & -0.60 & 0.73 & $\mathrm{j} 2$ & -0.53 & -0.46 & -0.31 & -0.92 & 1.34 \\
\hline e1 & -0.03 & -0.02 & -0.01 & -0.03 & 0.03 & j3 & -0.56 & -0.48 & -0.34 & -0.94 & 1.50 \\
\hline e2 & -0.20 & -0.12 & -0.06 & -0.21 & 0.22 & $\mathrm{j} 4$ & -0.45 & -0.30 & -0.18 & -0.56 & 0.68 \\
\hline e3 & -0.34 & -0.23 & -0.13 & -0.41 & 0.47 & $\mathrm{j} 5$ & -0.75 & -0.62 & -0.47 & -1.33 & 2.42 \\
\hline f1 & -0.20 & -0.13 & -0.07 & -0.22 & 0.24 & & & & & & \\
\hline $\mathrm{f} 2$ & -0.25 & -0.15 & -0.08 & -0.27 & 0.29 & & & & & & \\
\hline f3 & -0.20 & -0.12 & -0.06 & -0.21 & 0.22 & & & & & & \\
\hline $\mathrm{f} 4$ & -0.19 & -0.12 & -0.06 & -0.21 & 0.22 & & & & & & \\
\hline f5 & -0.19 & -0.12 & -0.06 & -0.21 & 0.22 & & & & & & \\
\hline
\end{tabular}



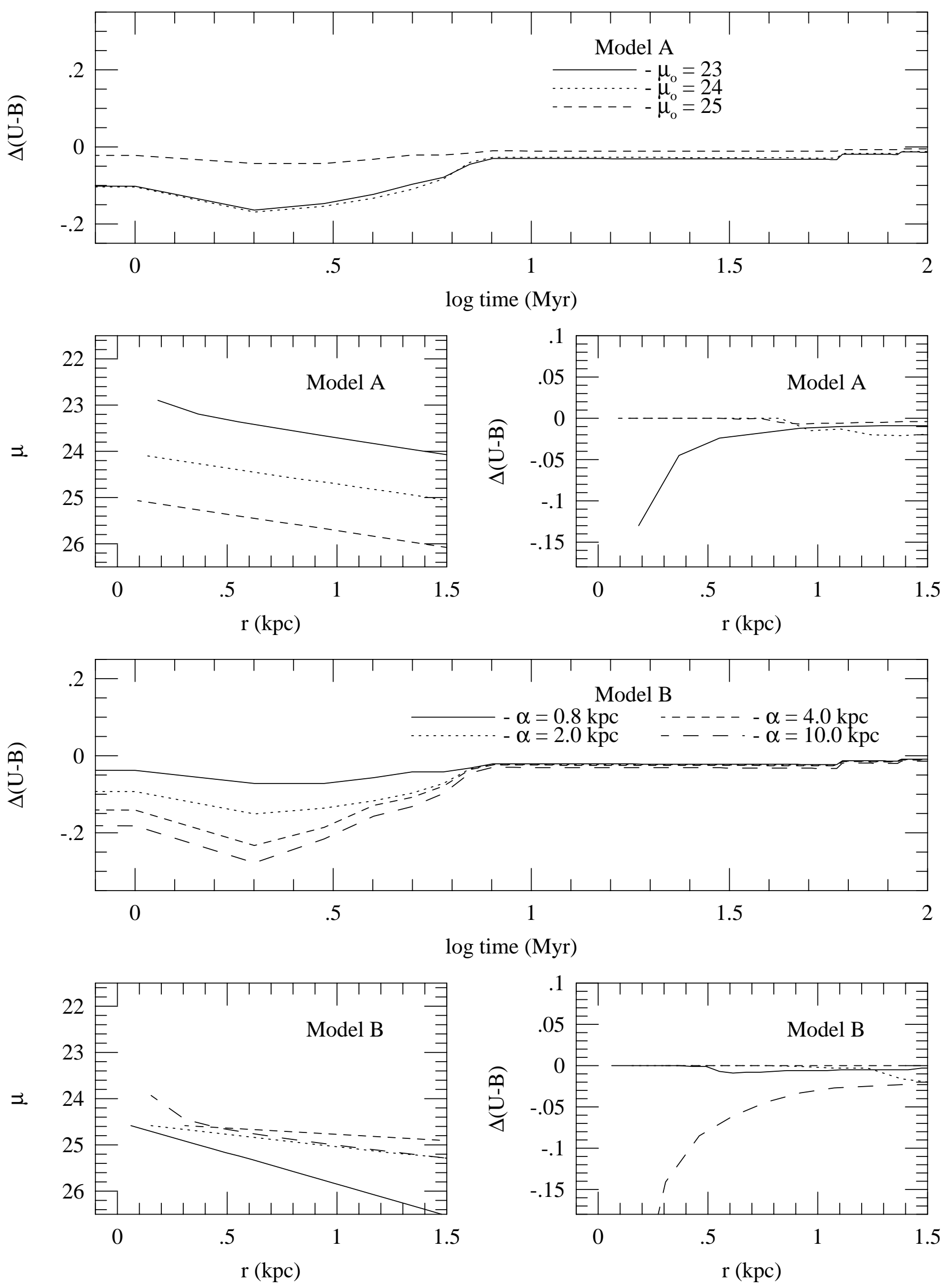

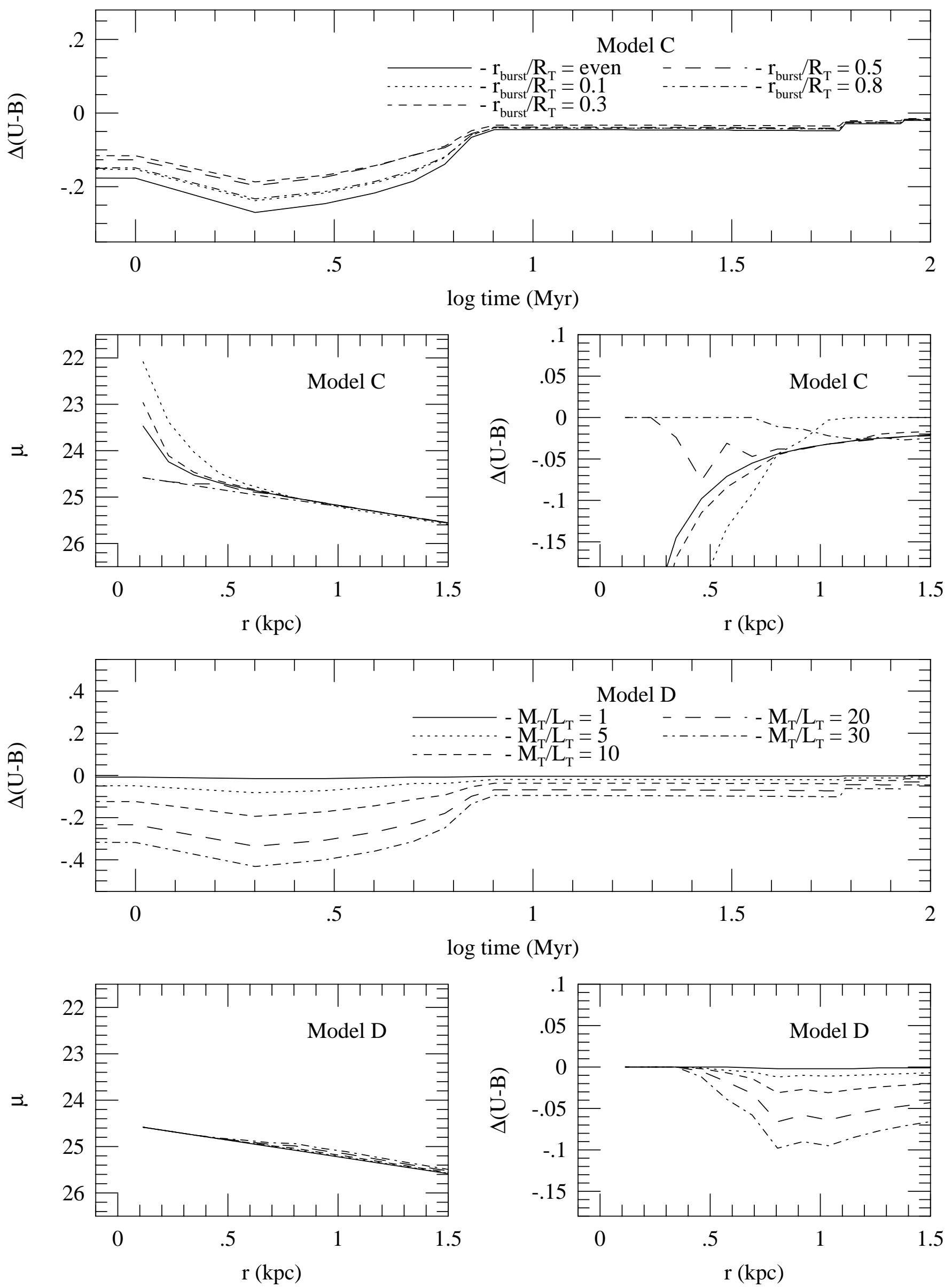

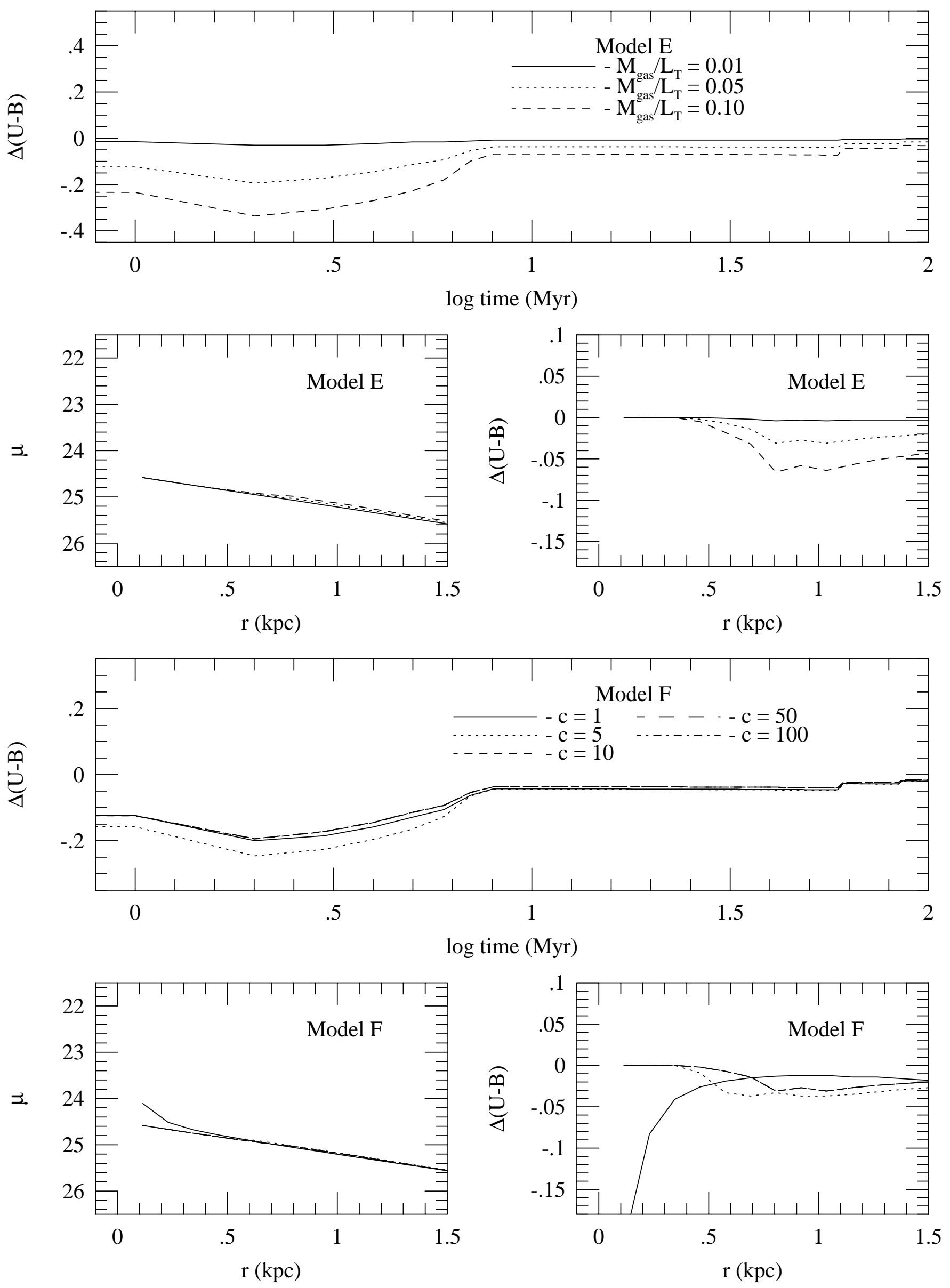

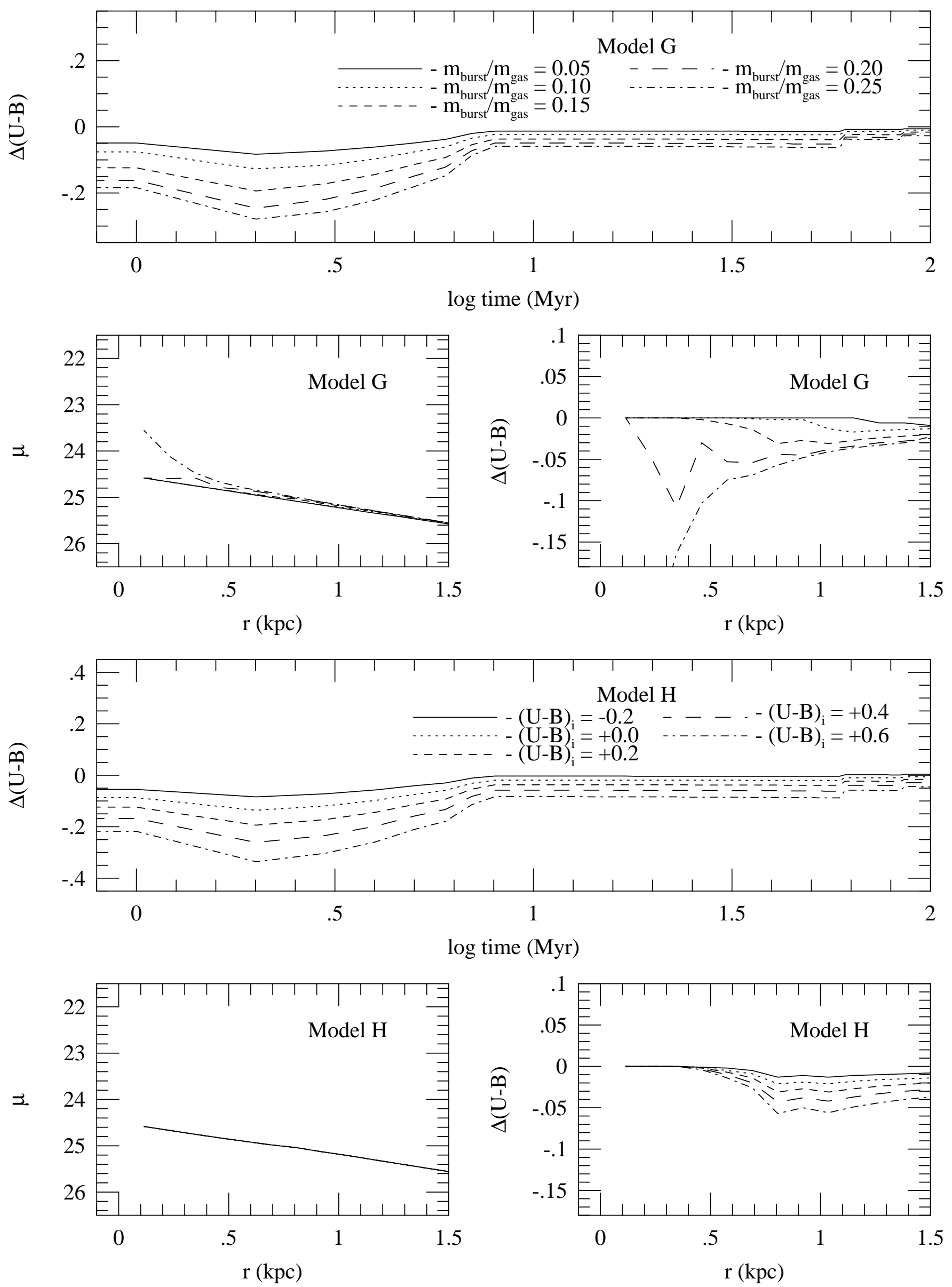

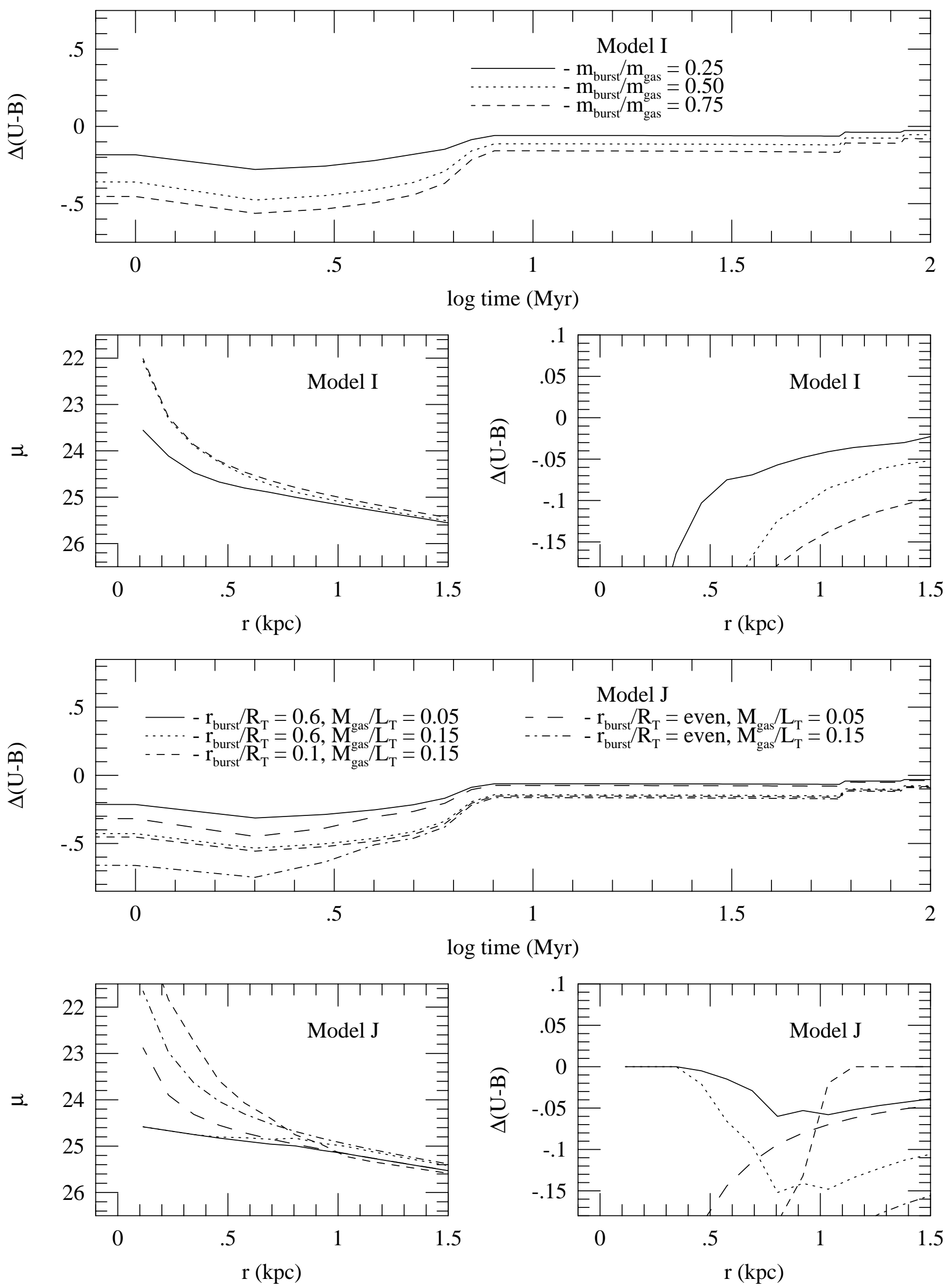

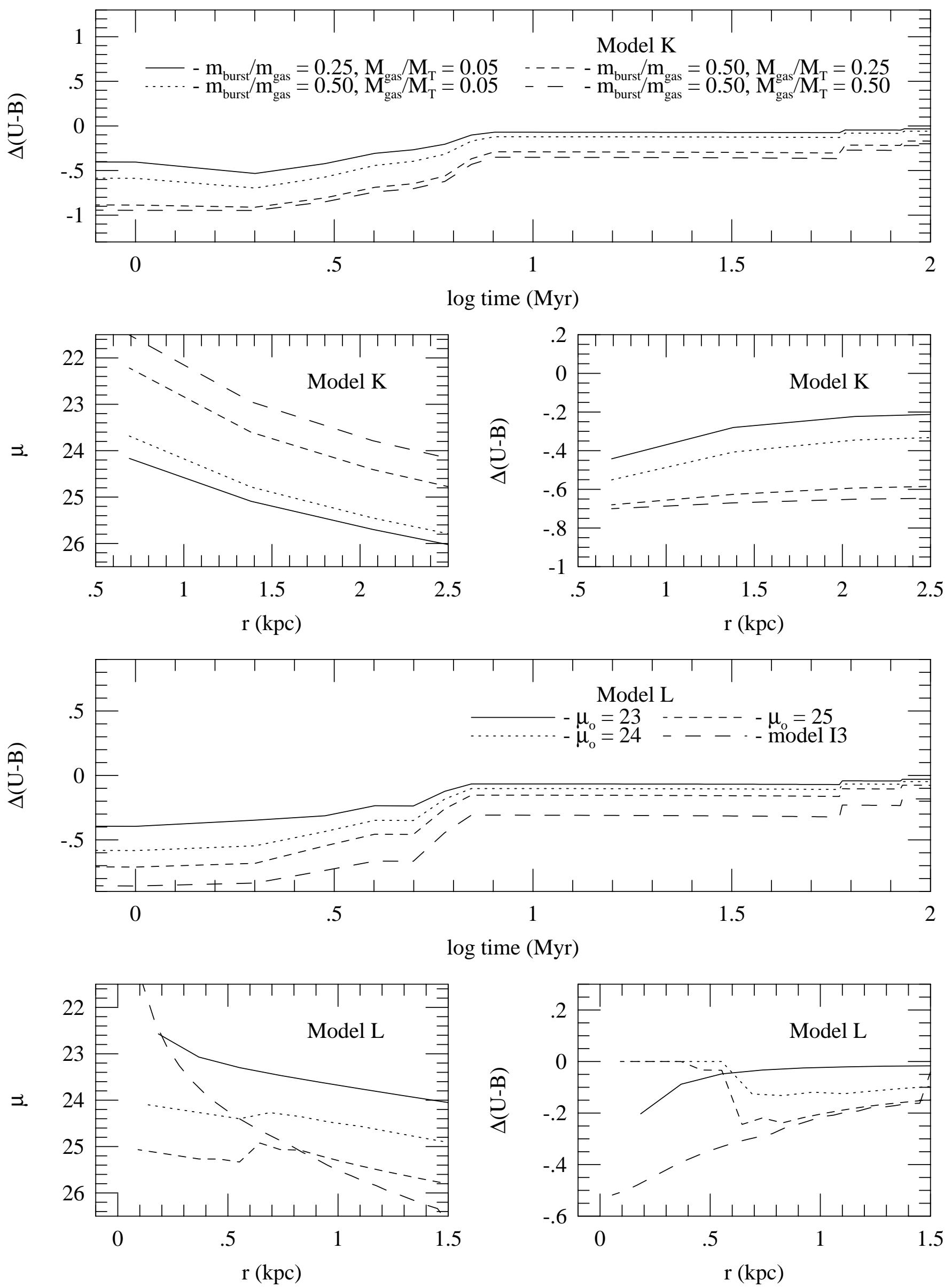
Table 4.

\begin{tabular}{|c|c|c|c|c|c|c|c|c|c|c|c|}
\hline Model & $\Delta(\mathrm{U}-\mathrm{B})$ & $\Delta(\mathrm{B}-\mathrm{V})$ & $\Delta(\mathrm{V}-\mathrm{I})$ & $\Delta \mathrm{B}_{\mathrm{mag}}$ & $\frac{\Delta \mathrm{L}}{\mathrm{I}}$ & Model & $\Delta(\mathrm{U}-\mathrm{B})$ & $\Delta(\mathrm{B}-\mathrm{V})$ & $\Delta(\mathrm{V}-\mathrm{I})$ & $\Delta \mathrm{B}_{\mathrm{mag}}$ & $\frac{\Delta \mathrm{L}}{\mathrm{L}}$ \\
\hline a1 & -0.16 & -0.10 & -0.05 & -0.18 & 0.17 & 11 & -0.35 & -0.23 & -0.13 & -0.41 & 0.46 \\
\hline $\mathrm{a} 2$ & -0.17 & -0.10 & -0.05 & -0.17 & 0.17 & 12 & -0.55 & -0.38 & -0.24 & -0.73 & 0.97 \\
\hline a3 & -0.04 & -0.03 & -0.01 & -0.05 & 0.04 & 13 & -0.68 & -0.53 & -0.38 & -1.11 & 1.79 \\
\hline i3 & -0.56 & -0.47 & -0.32 & -0.97 & 1.45 & 14 & -0.84 & -0.82 & -0.80 & -2.28 & 7.13 \\
\hline
\end{tabular}




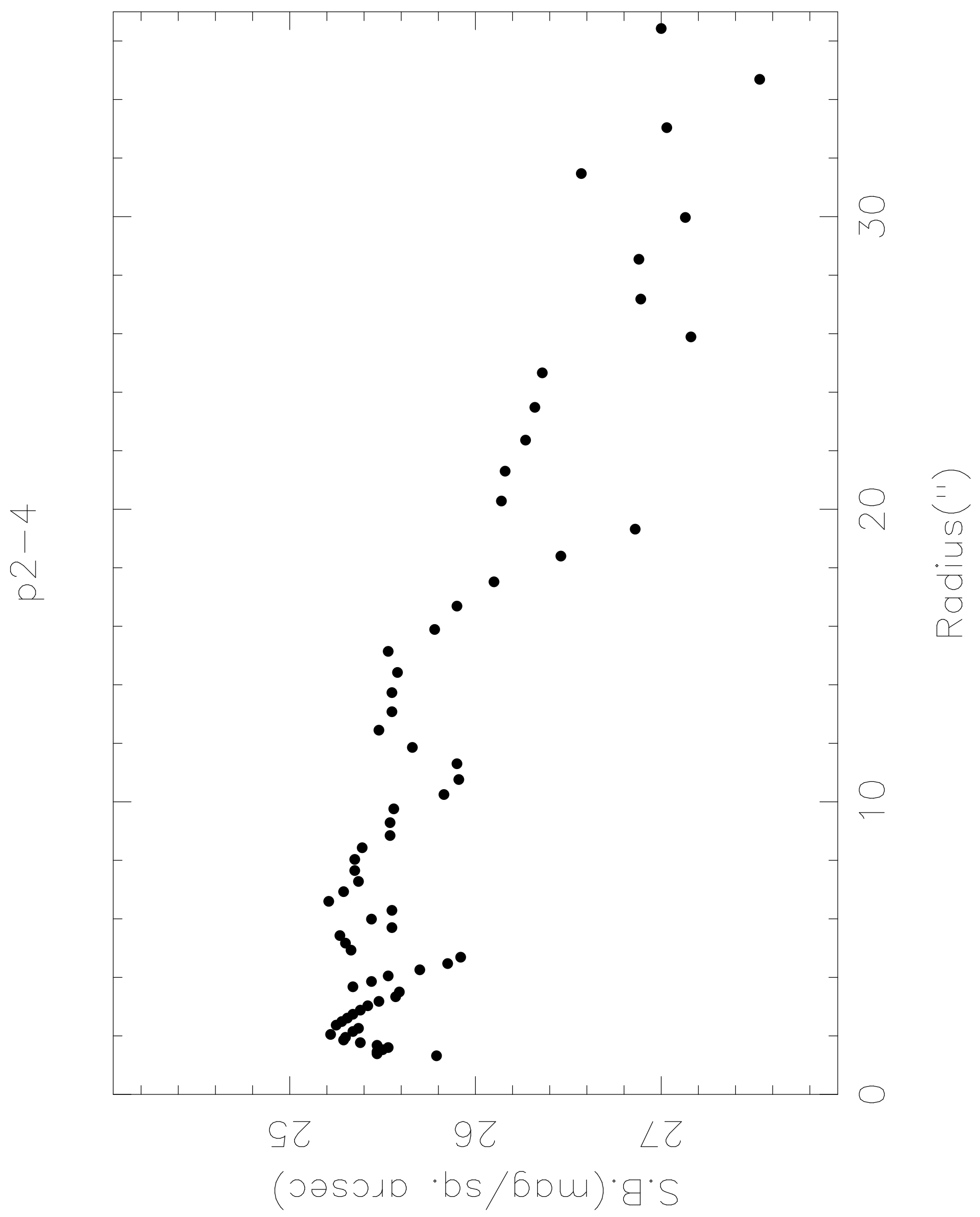




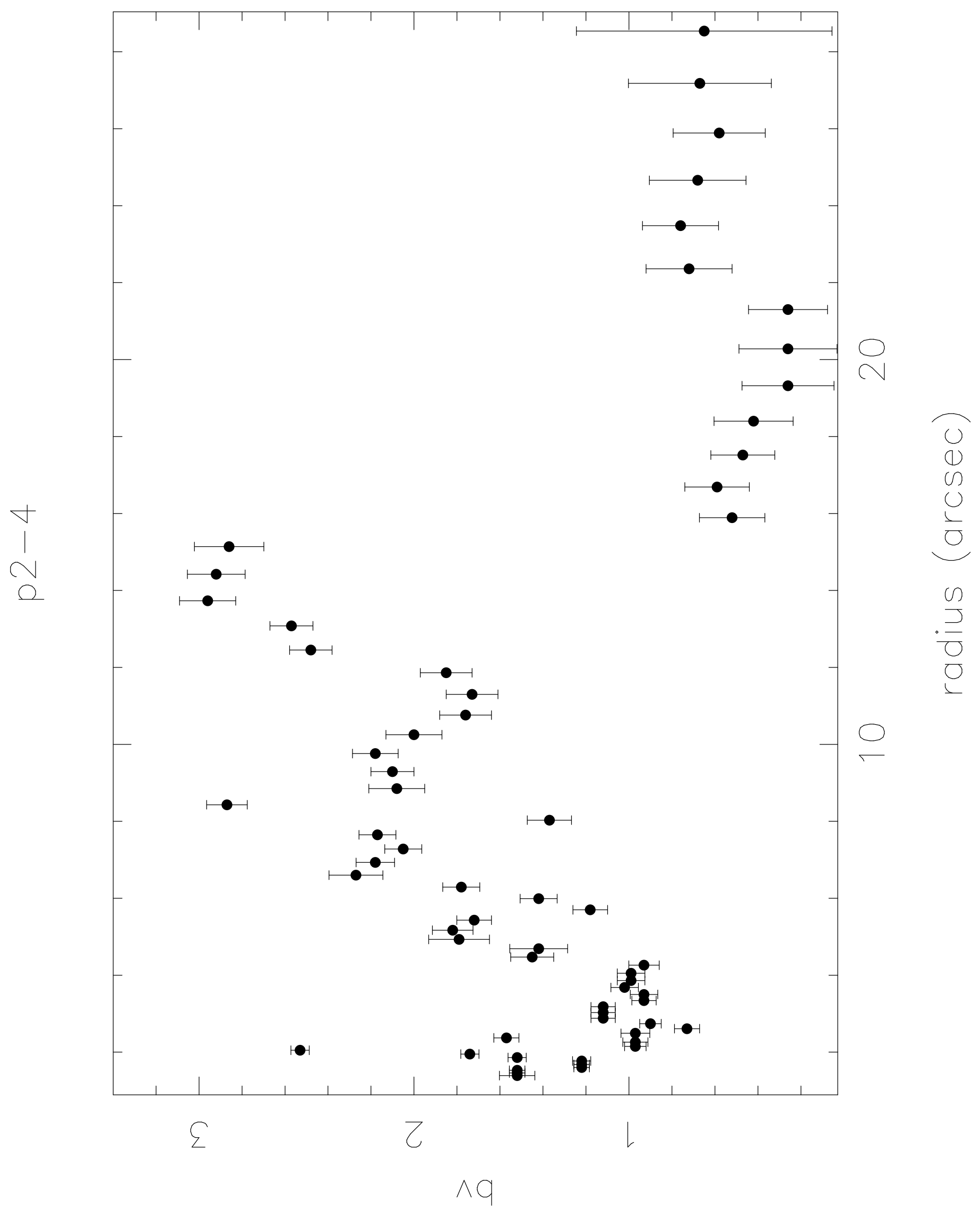




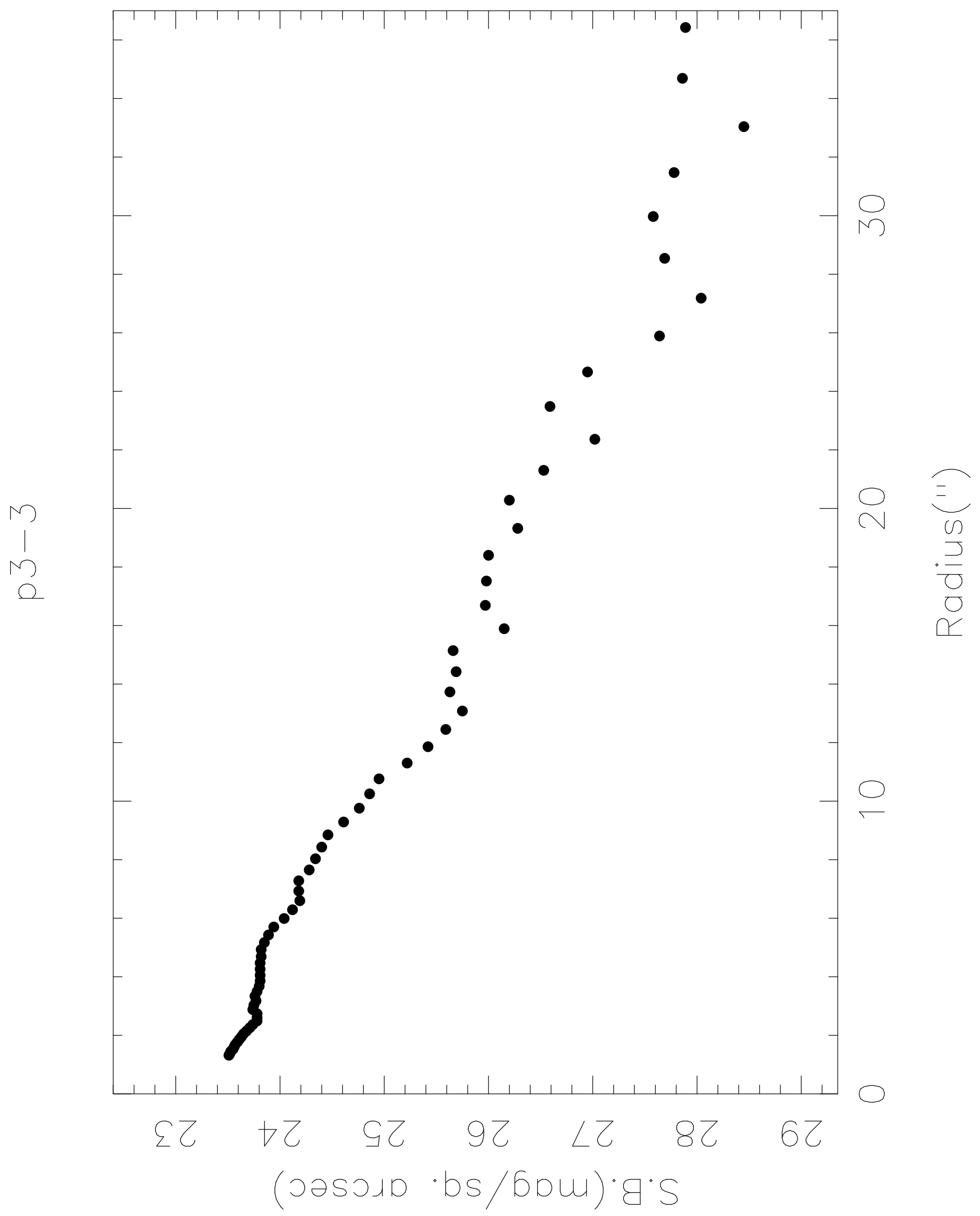




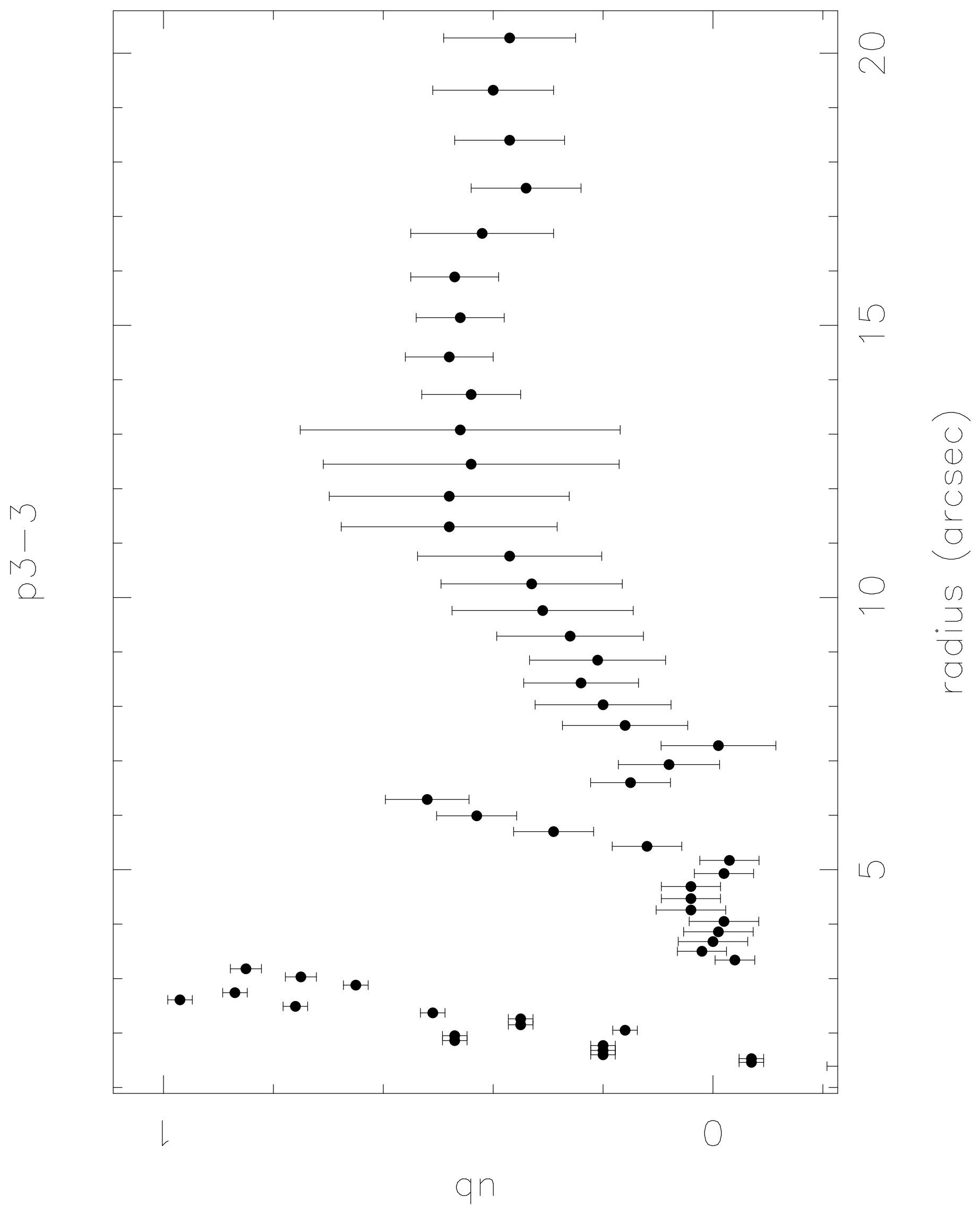

